\title{
Single-cell analysis of dihydroartemisinin-induced apoptosis through reactive oxygen species-mediated caspase-8 activation and mitochondrial pathway in ASTC-a-1 cells using fluorescence imaging techniques
}

\author{
Ying-Ying Lu \\ South China Normal University \\ Institute of Laser Life Science \\ MOE Key Laboratory of Laser Life Science \\ Guangzhou 510631, China
}

\author{
Tong-Sheng Chen \\ South China Normal University \\ Institute of Laser Life Science \\ MOE Key Laboratory of Laser Life Science \\ Guangzhou 510631, China \\ and \\ South China Normal University \\ Joint Laboratory of Laser Oncology with Cancer Center \\ of Sun Yat-Sen University \\ Guangzhou 510631, China
}

\section{Xiao-Ping Wang}

The First Affiliated Hospital of Jinan University Department of Anesthesiology Guangzhou 510631, China

\author{
Li Li \\ Laboratory of Laser Oncology with Cancer Center \\ of Sun Yat-Sen University \\ Guangzhou 510631, China
}

\begin{abstract}
Dihydroartemisinin (DHA), a front-line antimalarial herbal compound, has been shown to possess promising anticancer activity with low toxicity. We have previously reported that DHA induced caspase-3-dependent apoptosis in human lung adenocarcinoma cells. However, the cellular target and molecular mechanism of DHAinduced apoptosis is still poorly defined. We use confocal fluorescence microscopy imaging, fluorescence resonance energy transfer, and fluorescence recovery after photobleaching techniques to explore the roles of DHA-elicited reactive oxygen species (ROS) in the DHAinduced $\mathrm{BCl}-2$ family proteins activation, mitochondrial dysfunction, caspase cascade, and cell death. Cell Counting Kit- 8 assay and flow cytometry analysis showed that DHA induced ROS-mediated apoptosis. Confocal imaging analysis in a single living cell and Western blot assay showed that DHA triggered ROS-dependent Bax translocation, mitochondrial membrane depolarization, alteration of mitochondrial morphology, cytochrome $c$ release, caspase-9, caspase-8, and caspase- 3 activation, indicating the coexistence of ROS-mediated mitochondrial and death receptor pathway. Collectively, our findings demonstrate for the first time that DHA induces cell apoptosis by triggering ROS-mediated caspase-8/Bid activation and the mitochondrial pathway, which provides some novel insights into the application of DHA as a potential anticancer drug and a new therapeutic strategy by targeting ROS signaling in lung adenocarcinoma therapy in the future. $\odot 2010$ Society of Photo-Optical Instrumentation Engineers. [DOI: $10.1117 / 1.3481141]$
\end{abstract}

Keywords: dihydroartemisinin; reactive oxygen species; apoptosis; confocal imaging; fluorescence resonance energy transfer; fluorescence recovery after photobleaching; caspases; $\mathrm{BCl}-2$ family proteins.

Paper 10141R received Mar. 21, 2010; revised manuscript received Jun. 23, 2010; accepted for publication Jul. 1, 2010; published online Aug. 23, 2010.

\section{Introduction}

Dihydroartemisinin (DHA), a semisynthetic derivative of artemisinin, isolated from the traditional Chinese herb Artemisia annua, is recommended as a safe and effective mainstay in treating malaria by the World Health Organization (WHO). ${ }^{1}$ Many recent studies, including those from our laboratory, have revealed that DHA can inhibit the growth of cancer cells through an apoptotic pathway.,3 Activation of the cell death program (apoptosis) is a therapeutic approach for inhibiting malignant progression; thus, promoting apoptosis is a strategy for cancer drug discovery. ${ }^{4}$ Specifically, DHA-induced tumor cell apoptosis is implicated in causation of $\mathrm{G}_{0} / \mathrm{G}_{1}$ cell cycle arrest, ${ }^{5}$ activation of caspases ${ }^{3,5-8}$ and p38 kinase, ${ }^{9}$ decrease of $\mathrm{Bcl}-2 / \mathrm{Bax}$ expression ratio, ${ }^{5,6}$ and regulation of angiogenesis-

Address all correspondence to: Tongsheng Chen, South China Normal University, Institute of Laser Life Science, Guangzhou 510631, China. Tel: 86-2085211436-8613; Fax: 86-20-85216052; E-mail: chentsh@scnu.edu.cn related genes. ${ }^{10}$ Despite these advances, however, the cellular target and molecular mechanism of the action of DHA remain largely unclear.

The role of reactive oxygen species (ROS) as important mediators for the apoptotic signaling pathway is well supported, ${ }^{11}$ and high levels of ROS in cells are thought to be associated with a number of human diseases, especially cancer. ${ }^{12}$ Some reports showed that ROS generation during mitochondria-dependent apoptosis occurs downstream of the release of cytochrome $c$ from mitochondria to cytosol. ${ }^{13}$ In many apoptosis models, however, ROS act as upstream signaling molecules that initiate cell death. ${ }^{14,15}$ Members of the Bcl-2 family, including pro- and antiapoptotic proteins, are major regulators of mitochondrial-dependent apoptosis, ${ }^{16}$ and deregulation of $\mathrm{Bcl}-2$ family proteins has been implicated in the development of many malignancies. ${ }^{17} \mathrm{Bcl}-\mathrm{xL}$, a strongly

$1083-3668 / 2010 / 15(4) / 046028 / 16 / \$ 25.00$ @ 2010 SPIE 
antiapoptotic member of the Bcl-2 family, inhibits apoptosis induced by antineoplastic agents, improving the ability of tumor cells to escape from therapy. ${ }^{18}$ Several stimuli, such as sodium butyrate in mesothelioma cell lines ${ }^{19}$ and TGF- $\beta 1$ in the NRP-154 rat prostate adenocarcinoma cell line, ${ }^{20}$ induce apoptosis that can be correlated with substantial downregulation of Bcl-xL expression. Furthermore, it is considered that Bcl-xL inhibits membrane permeabilization and cytochrome $c$ release by blocking the activation of proapoptotic proteins $\mathrm{Bax}$ and $\mathrm{Bak}^{21} \mathrm{Bax}$, a proapoptotic $\mathrm{Bcl}-2$ family member, is a potent death executor that undergoes a conformational change and translocates from cytosol to the outer mitochondrial membrane in response to a variety of apoptotic signals in the early step of the mitochondrial apoptotic pathway. ${ }^{22}$ Several research groups have reported that ROS can act upstream of Bax translocation from cytosol into mitochondria, ${ }^{14,23}$ which leads to the loss of mitochondrial membrane potential $\left(\Delta \Psi_{m}\right)$, proapoptotic factors release, caspase cascade activation, and subsequent apoptosis induction. $^{22}$

The peroxide bridge in the DHA molecule may induce oxidative stress. Li et al. have shown that reaction of the endoperoxide bridge with heme irons leads to ROS generation and carbon-centered radicals. ${ }^{24}$ It has been reported that DHA-induced apoptosis is involved in ROS generation in Hela cells. ${ }^{7}$ Nevertheless, Lu et al. found that although DHA elicited superoxide anions, these ROS contributed little to DHA-induced apoptosis in HL-60 cells. ${ }^{9}$ Based on these notions, however, there has been no report so far to identify whether ROS act as key regulators in DHA-triggered apoptosis or whether ROS generation is simply a concomitant phenomenon in DHA-treated cells.

Fluorescence resonance energy transfer (FRET) and fluorescence recovery after photobleaching (FRAP) have been widely used to study protein-protein interactions and the mobility of protein molecules in living cells, ${ }^{25-30}$ respectively. To detect caspase- 9 and caspase- 3 activation in living cells, Takemoto et al. ${ }^{31}$ constructed SCAT9 and SCAT3 plasmids based on FRET and green fluorescent proteins (GFPs). SCAT9 or SCAT3 consists of a donor (enhanced cyan fluorescent protein, ECFP) and an acceptor (Venus, a mutant of yellow fluorescent protein, YFP). The donor and acceptor are linked with a caspase- 9 or caspase- 3 recognition and cleavage sequence (LEHD or DEVD). ${ }^{29,31}$ The activated caspase- 9 or caspase- 3 cleaves the linker LEHD or DEVD, which induces a marked decrease of FRET efficiency. Likewise, a fusion protein, YFPBid-CFP (FRET-Bid), which was constructed by connecting cyan fluorescent protein (CFP) and yellow fluorescent protein (YFP) to the $\mathrm{C}$ terminus and $\mathrm{N}$ terminus of Bid, respectively, has been used to monitor caspase- 8 activation in living cells. ${ }^{32}$

In this report, confocal fluorescence imaging, FRET, and FRAP have been used to analyze ROS generation, Bax translocation, caspases activation, mitochondrial fragmentation, loss of mitochondrial membrane potential $\left(\Delta \Psi_{m}\right)$, and cytochrome $c$ release inside single living cells. Our data suggest a previously unappreciated role for DHA-elicited ROS in regulating both mitochondrial and death receptor signaling pathways, which may function as a novel therapeutic strategy for the treatment of lung adenocarcinoma.

\section{Materials and Methods}

\subsection{Reagents}

DHA was obtained from Bide Pharmaceutical Corporation (Guangzhou, Guangdong Province, China). Working solutions were prepared by dissolving the compound in dimethyl sulphoxide (DMSO) before experiments. The final concentration of DMSO was less than $1 \%$ in all experiments. Lipofectamine 2000 and Mitotracker Red 633 were purchased from Invitrogen (Carlsbad, California). $\mathrm{N}$-acetyl cysteine (NAC), Rhodamine123 (Rho123), Hoechst 33258, propidium iodide (PI), RNase A, SP600125, and SB203580 were obtained from Sigma (St. Louis, Missouri). Cell Counting Kit-8 (CCK-8) was purchased from Dojindo Laboratories (Kumamoto, Japan). Annexin V-FITC apoptosis detection kit was obtained from Bender Medsystems (Vienna, Austria). Caspase-3 substrate (Ac-DEVD-AFC), caspase-8 substrate (Ac-IETDAFC), caspase-9 substrate (Ac-LEHD-AFC), staurosporine (STS), and U0126 were purchased from Alexis Biochemicals (Lausen, Switzerland). Mitochondria/cytosol fractionation kit was obtained from Calbiochem (Darmstadt, Germany). 2', 7'-Dichlorodihydrofluorescin diacetate (DCFH-DA) was purchased from Wako Ltd (Osaka, Japan). Anti-cytochrome $c$ antibodies were obtained from Santa Cruz Biotechnology (Santa Cruz, California). Anti-Bcl-xL, anti-Bax, anticaspase-3, anti- $\beta$-actin, anti-phospho-ERK, anti-ERK, antiphospho-JNK, anti-JNK, anti-phospho-p38, anti-p38 antibodies, and positive phospho-ERK/JNK/P38 control protein were all obtained from Cell Signaling (Beverly, Massachusetts). All the secondary antibodies were supplied by Molecular Probes (Eugene, Oregon).

\subsection{Cell Culture and Transfection}

The ASTC-a-1 cell line was obtained from the Department of Medicine, Jinan University (Guangzhou, China), and cultured in Dulbecco's modified Eagle's medium (DMEM; Gibco, Grand Island, New York) supplemented with $10 \%$ fetal calf serum (FCS; Sijiqing, Hangzhou, China), 50 units $/ \mathrm{ml}$ penicillin, and $50 \mu \mathrm{g} / \mathrm{ml}$ streptomycin in $5 \% \mathrm{CO}_{2}$ at $37{ }^{\circ} \mathrm{C}$ in a humidified incubator. For fluorescence studies, cells were transiently transfected with plasmids using lipofectamine 2000 in a $35-\mathrm{mm}$ dish for 24 to $48 \mathrm{~h}$. Cells stably expressing SCAT3 reporter were screened with $0.8 \mathrm{mg} / \mathrm{ml} \mathrm{G} 418$, and positive clones were picked up with micropipettes. ${ }^{29}$

\subsection{Cell Viability and Apoptosis Assay}

Cell viability was assessed by CCK-8 assay, according to the supplier recommendations. Viable cells were counted by absorbance measurements at $450 \mathrm{~nm}$ using an auto-microplate reader (infinite $\mathrm{M} 200$, Tecan, Austria). The $\mathrm{OD}_{450}$ value was proportional to the degree of cell viability. All experiments were performed in quadruple on three separate occasions.

Apoptosis of cells was examined by two approaches: Hoechst 33258 staining and flow cytometric (FCM) analysis using Annexin V-FITC/PI staining were performed as we previously described, ${ }^{3}$ and for each FCM analysis, 10,000 events were recorded. 


\subsection{Cell Cycle Analysis}

The proportions of cells in sub- $\mathrm{G}_{0}$ (apoptosis), $\mathrm{G}_{0} / \mathrm{G}_{1}$, $\mathrm{G}_{2} / \mathrm{M}$, and $\mathrm{S}$ phases were determined by FCM analysis of DNA content. To evaluate the cell cycle profile, cells (about $1 \times 10^{6}$ cells) were harvested, washed twice with PBS, and fixed in ice-cold $70 \%(\mathrm{v} / \mathrm{v})$ ethanol for $1 \mathrm{~h}$ at $4{ }^{\circ} \mathrm{C}$. Prior to analysis, samples were washed again and incubated in PBS containing $10 \mu \mathrm{g} / \mathrm{ml}$ RNase A for $30 \mathrm{~min}$, and then incubated with $5 \mu \mathrm{g} / \mathrm{ml} \mathrm{PI}$ at $37^{\circ} \mathrm{C}$ in the dark for $30 \mathrm{~min}$. DNA content was determined using an FCM (FACS, Arla BD, San Jose, California), and data were analyzed by FCS Express version 3 software. For each analysis, 10,000 events were recorded.

\subsection{Measurement of Intracellular ROS Generation}

ROS generation inside living cells was measured by confocal microscope imaging and FCM analysis using DCFH-DA, an oxidation-sensitive probe, which is cleaved intracellularly by nonspecific esterases and turns to highly fluorescent DCF upon oxidation by ROS. Untreated or treated cells were stained with $20 \mu \mathrm{M}$ DCFH-DA for $30 \mathrm{~min}$ in the dark and subsequently assayed by FACScan. For each analysis, 10,000 events were recorded.

\subsection{Determination of Mitochondrial Size}

The mitochondrial transversal section (width) is the most constant dimension of the organelle, and variations on this parameter reflect an alteration of the morphology of mitochondria. ${ }^{33}$ Cells were cultivated on a coverslip of a $35-\mathrm{mm}$ chamber. After desired treatment, the cells were washed with PBS three times and incubated with $0.1 \mu \mathrm{M}$ Mitotracker Red 633 for $30 \mathrm{~min}$ at room temperature in the dark. The cells were then washed three times with PBS and visualized under confocal microscope (LSM510/ConfoCor2, Zeiss, Jena, Germany). Mitotracker Red was excited at $633 \mathrm{~nm}$, and the emitted light was recorded through a 650-nm long-pass filter. For every condition tested, the size of 100 to 200 mitochondria in at least 50 different cells is from three independent experiments. The determination of the mitochondrial size was carried out using Zeiss Rel 3.2 image processing software (Zeiss, Jena, Germany) as we previously described. ${ }^{34}$

\subsection{Fluorescence Recovery after Photobleaching (FRAP) Analysis inside Single Living Cell}

FRAP analysis was performed on a confocal microscope (LSM510/ConfoCor2, Zeiss, Jena, Germany). Here, to investigate the mobility of GFP-Bax and the integrity of mitochondria after different treatments, the GFP or Mitotracker Red 633 in the indicating regions of living cells were photobleached by scanning the region with the maximal 488-nm or 633-nm laser line, and subsequently, the entire cell was imaged at every $5 \mathrm{~s}$ with a low laser power (5\% power) excitation for a duration of $500 \mathrm{~s}$ to monitor the recovery of fluorescence.

\subsection{Dynamic Monitoring of Bax Translocation and Cytochrome c Release}

A confocal laser scanning microscope (LSM510/ConfoCor2, Zeiss, Jena, Germany) was used to perform time-lapse fluo- rescence imaging of Bax translocation and cytochrome $c$ release inside single living cells. Thirty-six hours after transiently expressing GFP-Bax or GFP-cytochrome $c$ (GFP-Cyt.c) and DsRed-Mito, cells were treated and then imaged by the confocal microscope at $37^{\circ} \mathrm{C}$. The images of cells expressing GFP-Bax or GFP-Cyt.c and DsRed-Mito were collected using dual fluorescence channels. The excitation wavelengths were $488 \mathrm{~nm}$ for GFP and $543 \mathrm{~nm}$ for DsRed. The emission fluorescence channels were 500 to $550 \mathrm{~nm}$ for GFP and 600 to $650 \mathrm{~nm}$ for DsRed.

\subsection{Measurement of Mitochondrial Membrane Potential $\left(\Delta \Psi_{m}\right)$}

Rho123, a potential-sensitive dye, was used to evaluate changes in $\Delta \Psi_{m}$ by FCM as previously described. ${ }^{3}$ Briefly, cells after treatment with DHA for 0 and $24 \mathrm{~h}$ in the presence or absence of NAC were harvested and stained by $1 \mu \mathrm{M}$ Rho123 for $30 \mathrm{~min}$ at $37{ }^{\circ} \mathrm{C}$ in the dark. Cells were then washed with PBS twice and subsequently assayed by FACScan. Results were expressed as the proportion of cells with lost or low $\Delta \Psi_{m}$, which was estimated by reduced fluorescence intensity from Rho123. For each analysis, 10,000 events were recorded.

The $\Delta \Psi_{m}$ in single living cells was also monitored in realtime by time-lapse confocal imaging, as previously described. ${ }^{3}$ Briefly, the fluorescence images of cells stained with $5 \mu \mathrm{M}$ Rho123 were monitored in real time using a confocal microscope (LSM510/ConfoCor2, Zeiss, Jena, Germany) equipped with a device sustained culture condition $\left(37{ }^{\circ} \mathrm{C}, 5 \% \mathrm{CO}_{2}\right)$.

\subsection{Detection of Emission Spectra of SCAT3 inside Living Cells}

The cell line stably expressing SCAT3 was used for the emission spectral analysis of caspase- 3 activation inside living cells, as described in our previous work. ${ }^{3,34}$ Each well of a 96-well plate contained $5 \times 10^{5}$ cells stably expressing SCAT3. The emission spectra of SCAT3 inside living cells were scanned using an auto-microplate reader (Infinite M200, Tecan, Austria). The excitation wavelength was $430 \mathrm{~nm}$, and the scanning range of emission fluorescence was from $456 \mathrm{~nm}$ to $600 \mathrm{~nm}$. The scanning step was $2 \mathrm{~nm}$.

\subsection{Confocal Imaging and Fluorescence Resonance Energy Transfer (FRET) Acceptor Photobleaching Technique}

In this study, confocal imaging and FRET analysis were performed on a confocal microscope (LSM510/ConfoCor2, Zeiss, Jena, Germany). All the quantitative analysis of the fluorescence images was performed by Zeiss Rel 3.2 image processing software (Zeiss, Jena, Germany). For time-lapse imaging, culture dishes were mounted onto the microscope stage equipped with a temperature-controlled chamber (Zeiss, Jena, Germany).

For photobleaching acceptor studies, 458-nm laser from an argon ion laser was used for CFP excitation. Images were acquired through CFP and YFP filter channels, respectively. Here, the filter sets used were CFP (bandpass BP 470 to $500 \mathrm{~nm}$ ) and YFP (long-pass LP $530 \mathrm{~nm}$ ). 514-nm laser of an argon ion laser was used to photobleach the accep- 
tor of SCAT9 and FRET-Bid, respectively. In order to quantitatively analyze the FRET efficiency of FRET plasmids, we defined two parameters: $\Delta I_{C F P}$ and $\Delta I_{Y F P}$, where $\Delta I_{C F P}$ is the increase of CFP intensity, and $\Delta I_{Y F P}$ is the decrease of YFP intensity, as described in our previous report. ${ }^{35}$ The condition $\left(37{ }^{\circ} \mathrm{C}, 5 \% \mathrm{CO}_{2}\right)$ was sustained during measurement. All the quantitative analysis of the fluorescence images was performed by Zeiss Rel3.2 image processing software.

\subsection{Caspase Enzymatic Activities}

Activities of caspase-3, caspase-8, and caspase-9 were measured using fluorogenic substrates Ac-DEVD-AFC, Ac-IETDAFC, and Ac-LEHD-AFC, respectively, according to the manufacturer's instructions. PBS-washed cell pellets (derived from either the medium or the adherent cells) were resuspended in extract buffer [25 mM HEPES (pH7.4), $0.1 \%$ TritonX-100, 10\% glycerol, $5 \mathrm{mM}$ DTT, $1 \mathrm{mM}$ phenylmethylsulfonyl fluoride, $10 \mathrm{mg} / \mathrm{ml}$ pepstatin, and $10 \mathrm{mg} / \mathrm{ml} \mathrm{Leu-}$ peptin] and vortexed vigorously. A proluminescence caspase- 3 , caspase- 8 , or caspase- 9 substrate was added to the extract in each well of a 96 -well plate at $100 \mu \mathrm{M}$ final concentration for $2 \mathrm{~h}$ at $37{ }^{\circ} \mathrm{C}$, and caspase activity was measured continuously by monitoring the release of fluorigenic AFC using an auto-microplate reader (Infinite M200, Tecan, Austria). Caspase-like activity is reported as the ratio of the fluorescence output in treated samples relative to untreated controls.

\subsection{Protein Extraction and Western Blot Analysis}

After different treatments, ASTC-a-1 cells were washed twice in cold PBS and lysed in a buffer containing $50 \mathrm{mM}$ Tris $\mathrm{HCl}$ $\mathrm{pH}$ 8.0, $150 \mathrm{mM} \mathrm{NaCl}, 1 \%$ TritonX-100, $100 \mu \mathrm{g} / \mathrm{ml}$ PMSF and Protease Inhibitor Cocktail Set I (Calbiochem, San Diego, California). Lysates were separated by SDS-PAGE and transferred to a nitrocellulose membrane (Roch). The membrane was first blocked with 5\% skim milk for 0.5 to $1 \mathrm{~h}$, followed by incubation with primary antibodies overnight at $4{ }^{\circ} \mathrm{C}$. Primary antibody incubation was performed in $5 \%$ bovine serum albumin in TBS-T (Tris base saline, $\mathrm{pH} 7.4,0.1 \%$ Tween 20). A designated secondary antibody was diluted at the recommended ratio in TBS-T and 5\% bovine serum albumin for 2.0 to $3.0 \mathrm{~h}$ at room temperature. Washing with TBS-T was performed between all steps. Detection was performed using the Odyssey Scanning Infrared Fluorescence Imaging System (LI-COR, Lincoln, Nebraska).

\subsection{Statistical Analysis}

Results were expressed as mean \pm standard deviation (SD). A student's $t$-test was used to compare the mean differences between samples using the statistical software SPSS, version 10.0 (SPSS, Chicago). Throughout the work, $P$ values less than 0.05 were considered to be statistically significant. All charts were drawn using Origin software, version 6.0.

\section{Results}

\subsection{DHA-Induced ROS-Dependent Cytotoxicity in ASTC-a-1 Cells}

Our previous study showed that DHA effectively exerted cytotoxicity on ASTC-a-1 cells in a time- and concentration-

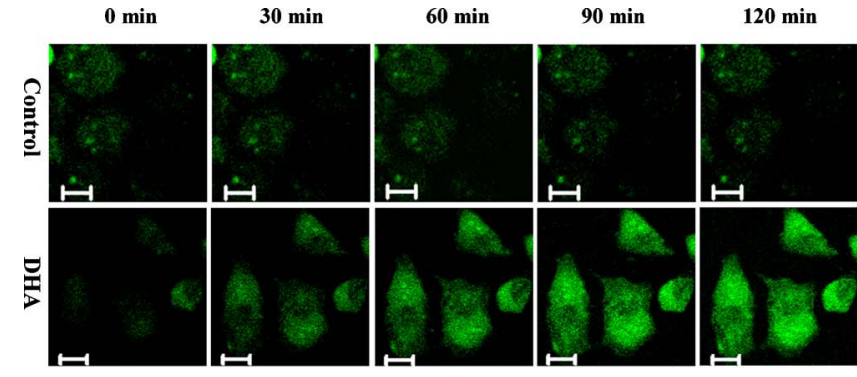

(a)

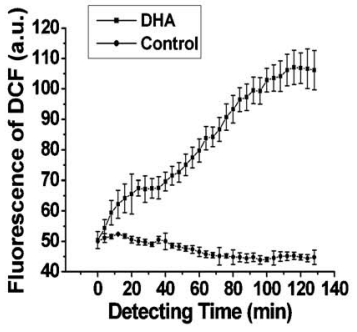

(b)

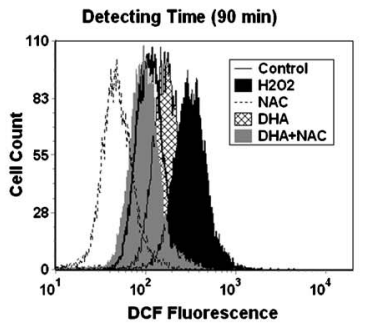

(c)

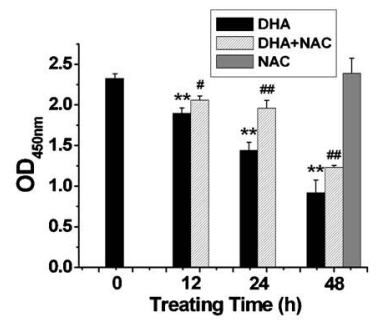

(d)

Fig. 1 DHA-induced ROS-dependent cytotoxicity in ASTC-a-1 cells. (a) Dynamical fluorescence images of DHA-induced ROS generation in living cells. Cells were incubated with $20 \mu \mathrm{M}$ DCFH-DA, an oxidation-sensitive fluorescent probe, for $30 \mathrm{~min}$ in the dark then treated with $20 \mu \mathrm{g} / \mathrm{ml} \mathrm{DHA}$. The levels of intracellular ROS were determined by the fluorescence of DCF inside living cells using a confocal microscope. Scale bar: $10 \mu \mathrm{m}$. (b) Mean traces of the dynamics of the DCF fluorescence corresponding to Fig. 1(a). (c) FCM analysis of DHA-elicited ROS under different conditions indicated. Untreated or treated cells were harvested and stained with $20 \mu \mathrm{M}$ DCFH-DA for 30 min before being subjected to FCM. Control: $\mathrm{H}_{2} \mathrm{O}_{2}$ treatment as a positive control. (d) Effect of NAC on DHA-induced cytotoxicity assessed by CCK-8. Cells were treated with $20 \mu \mathrm{g} / \mathrm{ml}$ DHA for $0,12,24$, and $48 \mathrm{~h}$ in the presence or absence of $5 \mathrm{mM}$ NAC. Experiments were conducted in quadruple wells, and data analyzed with SPSS 10.0 software were expressed as mean \pm SD. ${ }^{* *} P<0.01$, compared with control; ${ }^{\#} P<0.05$, ${ }^{\# \#} P<0.01$, compared with DHA treatment alone.

dependent manner. ${ }^{3}$ For every experiment described here, a lethal concentration of $20 \mu \mathrm{g} / \mathrm{ml}$ was used. In the present study, we used the same cell line to test the hypothesis that DHA-induced cell death is initiated by ROS. Intracellular ROS generation detected by DCFH-DA, an oxidationsensitive fluorescent probe, was assessed by confocal microscope imaging and FCM analysis for both control and DHAtreated cells. As can be seen in Fig. 1(a), exposure of cells to DHA elicited a rapid elevation of cellular ROS level within $30 \mathrm{~min}$, and the dynamics of DCF fluorescence corresponding 
to Fig. 1(a) are shown in Fig. 1(b). The consistent results from FCM analysis demonstrated that DHA-treated cells exhibited a marked increase of DCF fluorescence as $\mathrm{H}_{2} \mathrm{O}_{2}$ treatment (as a positive control), which was completely abrogated by the antioxidant NAC pretreatment [Fig. 1(c)], suggesting that DHA treatment led to a rapid induction of ROS inside cells. Moreover, CCK-8 assay was performed to further investigate the contribution of ROS to DHA-induced cytotoxicity, and our results indicated that NAC significantly attenuated DHAinduced cytotoxicity [Fig. 1(d)], suggesting that DHA-elicited ROS were essential for DHA to exert its cytotoxic activity.

\subsection{ROS Involvement in DHA-Triggered $\mathrm{G}_{2} / \mathrm{M}$ Arrest and Subsequent Apoptosis}

It has been suggested that the effects of ROS on initiating checkpoint responses influence the cell cycle progression. ${ }^{36}$ In this study, evaluation of DNA content by FCM analysis of PI staining demonstrated that the proportion of cells with sub- $\mathrm{G}_{0}$ DNA content, indicative of apoptotic cells, increased from $1.94 \%$ (control) to $14.4 \%$ and $34.0 \%$ at 24 and $48 \mathrm{~h}$ after DHA treatment, which was markedly attenuated by NAC pretreatment [Fig. 2(a)]. As shown in Fig. 2(b), DHA-treated cells exhibited time-dependent increases in the proportion of cells in $\mathrm{G}_{2} / \mathrm{M}$ phase, which also remarkably decreased in the case of pretreatment of NAC, indicating that DHA-elicited ROS played an important role in DHA-induced $\mathrm{G}_{2} / \mathrm{M}$ arrest.

In our previous study, DHA-provoked caspase-3dependent apoptosis was confirmed. ${ }^{3}$ We next sought to determine whether ROS were implicated in DHA-induced cell apoptosis. Hoechst 33258 staining experiments showed that chromatin was evenly present in the whole nucleus in control cells, and cells after exposure to DHA for $24 \mathrm{~h}$ underwent typical apoptotic morphology changes such as chromatin condensation, margination, and shrunken nucleus, which were effectively blocked by NAC pretreatment [Fig. 2(c)]. These results implied that DHA induced ROS-dependent cell death in an apoptotic fashion. Moreover, the early apoptotic characteristic of appearance of phosphatidyl serine (PS) on the extracellular side of the cell membrane was quantified by annexin V-FITC/PI staining. The consistent results from Fig. 2(d) showed that NAC pretreatment conferred protection against DHA-induced apoptosis, further demonstrating that DHA triggered ROS-mediated apoptosis.

\subsection{DHA-Induced ROS-Dependent Bax Translocation and Activation}

In some models of mitochondrial-dependent apoptosis, ROS act upstream to cause the activation of Bax or Bak. ${ }^{14}$ Here, we addressed the question of whether ROS acted upstream of Bax translocation and activation in DHA-induced apoptotic process. The FRAP technique was used to measure the mobility of the GFP-Bax fusion protein in single living cells after different treatments. Strikingly, we noticed a rapid refilling of GFP-Bax in the photobleached area for control cells [Fig. 3(a), upper panel], implying that GFP-Bax distributed freely throughout the cell. However, DHA treatment induced GFPBax puncta formation, in which the fluorescence recovery in the photobleached area was completely blocked [Fig. 3(a), middle panel], implying that DHA induced Bax activation and translocation. Upon pretreatment with NAC, the reoccurrence of fluorescence recovery was observed as a control cell [Fig. 3(a), lower panel], suggesting that ROS mediated the DHAinduced Bax translocation. Dynamics of FRAP from 15 to 20 cells in three independent experiments for control, DHAtreated, and DHA- and NAC-cotreated cells are shown in Fig. 3(b). Furthermore, confocal images and statistical results also confirmed that DHA triggered time-dependent Bax translocation, which was effectively inhibited by NAC pretreatment [Figs. 3(c) and 3(d)]. Based on these data, we concluded that ROS-mediated Bax recruitment after DHA treatment irreversibly localized to certain organelle membranes such as mitochondria or endoplasmic reticulum.

To determine whether DHA induced Bax translocation into mitochondria, we next monitored the dynamics of Bax translocation inside single living cells co-expressing GFP-Bax and DsRed-Mito using time-lapse confocal fluorescence microscope. We found that the diffuse distribution of GFP-Bax, after DHA treatment, became punctuated and colocalized with mitochondria, as visualized by a mitochondrial marker, DsRed-Mito [Fig. 3(e)]. The increase of relative fluorescence intensity of GFP-Bax in the enriched mitochondrial area (indicated as a white square) corresponding to Fig. 3(e) also confirmed that translocation of cytosolic Bax to mitochondrial membrane occurred during DHA-induced apoptotic cell death [Fig. 3(f)]. Last, Western blot analysis further demonstrated that DHA-provoked Bax translocation into mitochondria was significantly prevented by NAC pretreatment [Fig. 3(g)]. Collectively, these findings suggested that DHA-elicited ROS promoted Bax activation and translocation into mitochondria.

\subsection{ROS-Mediated Mitochondrial Dysfunction during DHA-Induced Apoptosis}

Mitochondrial dysfunction - in particular, the induction of the mitochondrial membrane potential $\left(\Delta \Psi_{m}\right)$ depolarization and ultrastructural changes - has been implicated in the cascade of events involved in the induction of apoptosis via the mitochondrial pathway. ${ }^{37}$ In this study, the dynamical loss of $\Delta \Psi_{m}$ in single living cells stained with potential-sensitive dye Rho123 was monitored by imaging the reduction of Rho123 fluorescence using a time-lapse confocal microscope. The time-lapse images of cells stained with Rho123 and the mean dynamical traces of fluorescence intensity of these three cells under different treatments are shown in Figs. 4(a) and 4(b), respectively. We found that compared with control cells, DHA treatment induced a significant decrease of Rho123 fluorescence, which was almost completely blocked by NAC pretreatment. Moreover, we used FCM to further confirm the loss of $\Delta \Psi_{m}$ by measuring the fluorescence of Rho123 under various treatments. At $24 \mathrm{~h}$ after DHA treatment, the percentage of cells with lost or low Rho123 fluorescence increased from $3.8 \%$ (control) to $38.8 \%$, which reduced to $21.5 \%$ in the presence of NAC [Fig. 4(c)]. These results revealed that DHA induced ROS-dependent loss of $\Delta \Psi_{m}$.

Recent studies have indicated that the loss of $\Delta \Psi_{m}$ leads to mitochondrial swelling, which is often associated with cell injury. ${ }^{38}$ Thus, we measured mitochondrial width by imaging the mitochondria stained with Mitotracker Red 633 inside living cells. Figure 4(d) represents the typical confocal images of mitochondrial morphology, displaying that in contrast to the normal long tubular mitochondria in control cells [Fig. 4(d), 


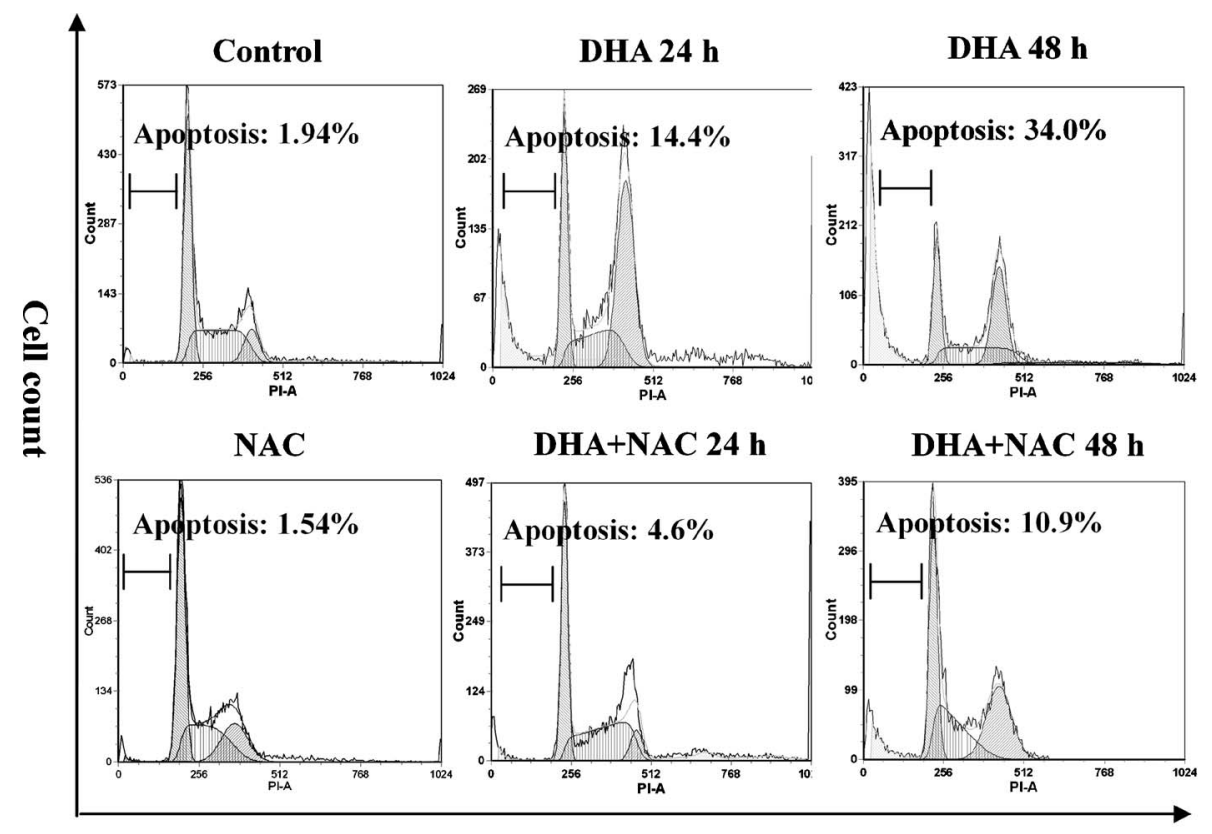

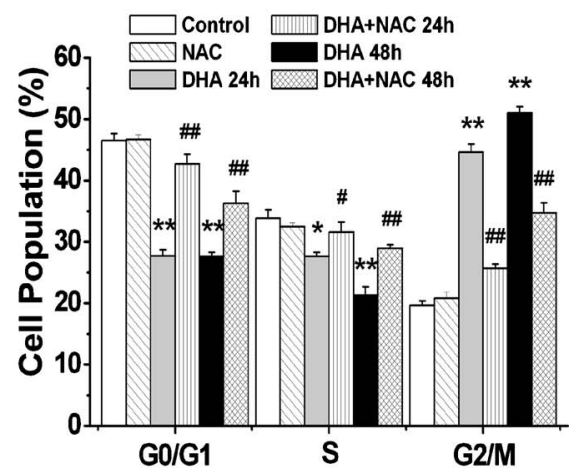

(b)

PI

(a)

Control

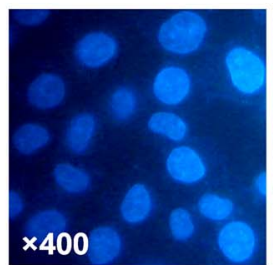

NAC 24 h

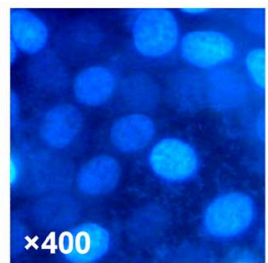

DHA 24 h

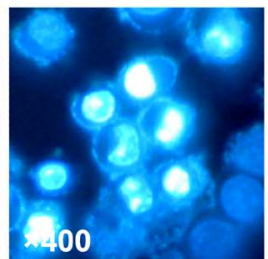

DHA+NAC 24 h

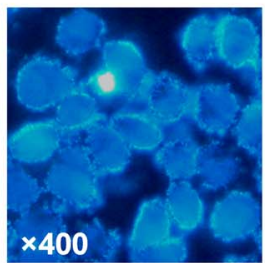

(c)

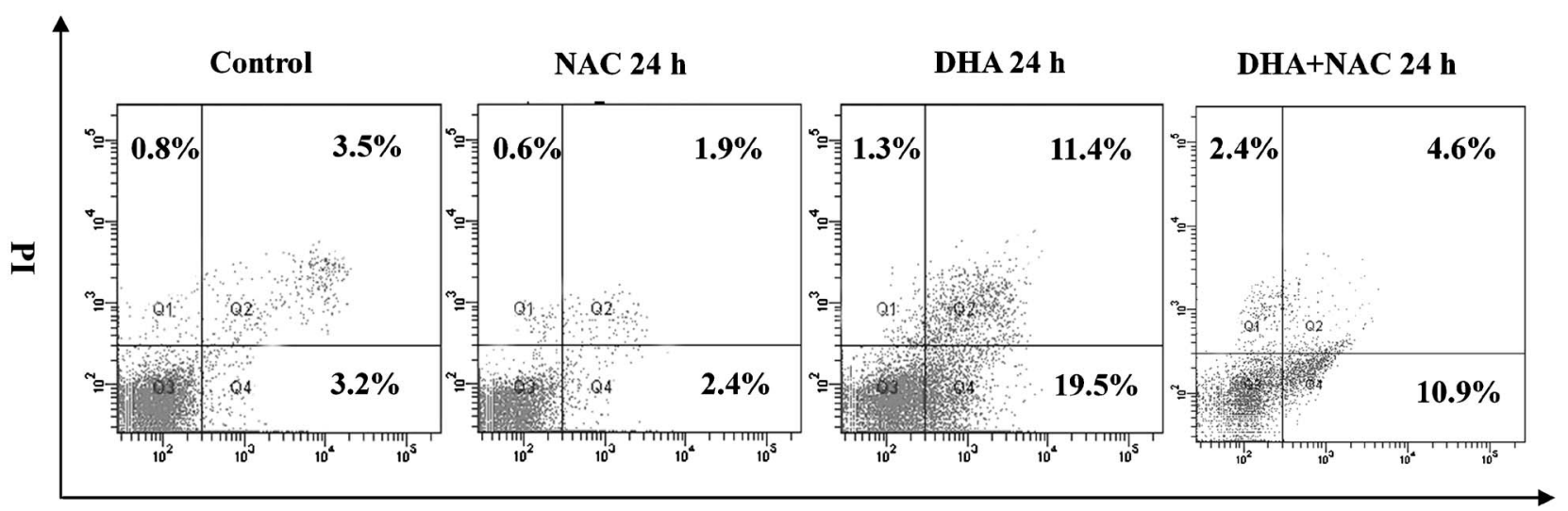

Annexin V-FITC

(d)

Fig. 2 DHA-induced ROS-mediated cell cycle arrest and apoptosis. (a) Determination of cell cycle profile by FCM. Cells were cultured with $20 \mu \mathrm{g} / \mathrm{ml} \mathrm{DHA}$ for 0,24 , or $48 \mathrm{~h}$ with or without the addition of $5 \mathrm{mM} \mathrm{NAC}$ and then stained with $5 \mu \mathrm{g} / \mathrm{ml}$ PI before being analyzed by FCM. (b) The proportion of cells in the $G_{0} / G_{1}, S$, and $G_{2} / M$ phases of the cell cycle were calculated using FCS express $V 3$ software and shown as mean $\pm S D$ from three independent experiments. ${ }^{*} P<0.05,{ }^{* *} P<0.01$, compared with control; ${ }^{\#} P<0.05,{ }^{\# \#} P<0.01$, compared with DHA treatment alone. (c) Nuclear morphology for apoptosis assessed by Hoechst 33258 staining. Images were recorded using a digital camera with $1280 \times 1280$ pixels resolution. Magnification 400. (d) FCM analysis of DHA-induced ROS-dependent apoptosis. Cells were treated with $20 \mu \mathrm{g} / \mathrm{ml} \mathrm{DHA}$ for 0 and $24 \mathrm{~h}$ in the presence or absence of $5 \mathrm{mM} \mathrm{NAC}$, stained with Annexin V-FITC/PI, and then analyzed by FCM. 


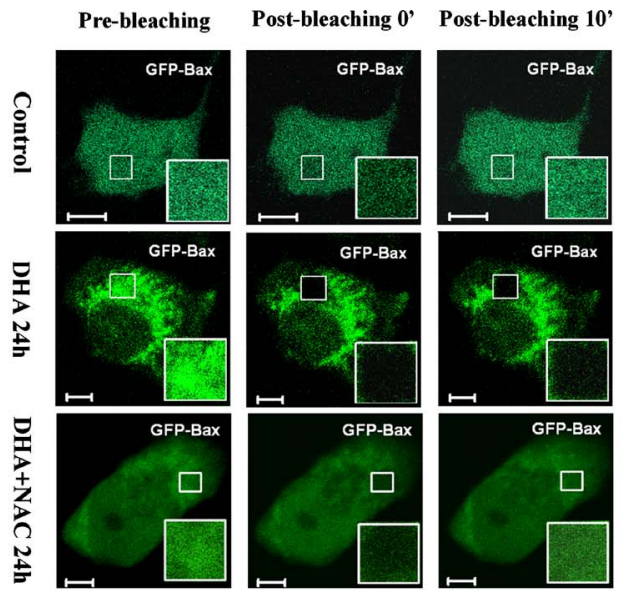

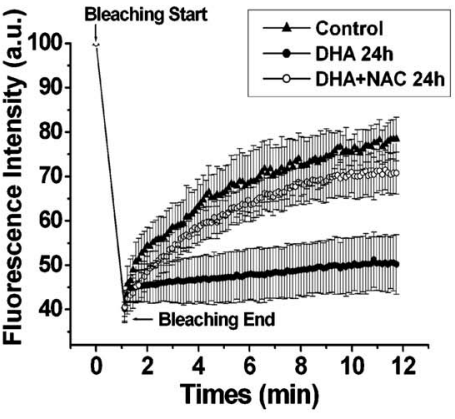

(b)

(a)

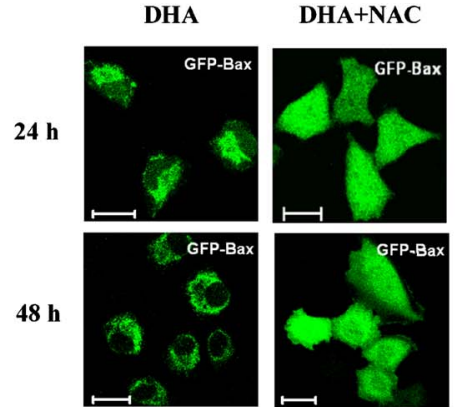

(c)
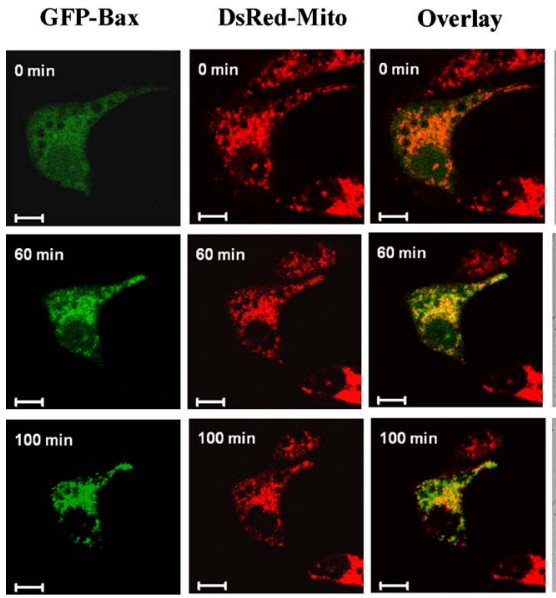

(e)
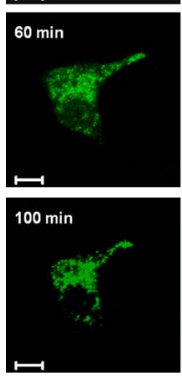

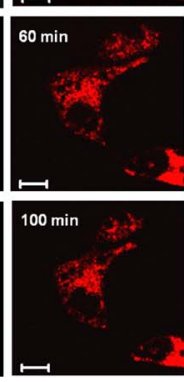

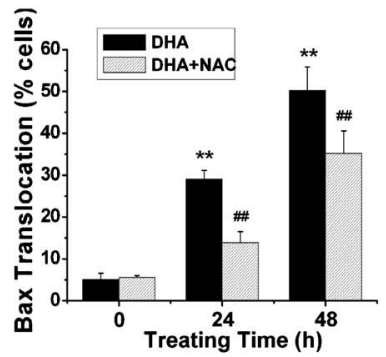

(d)

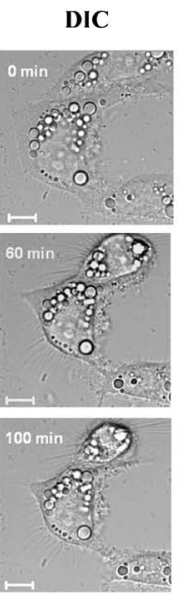

..

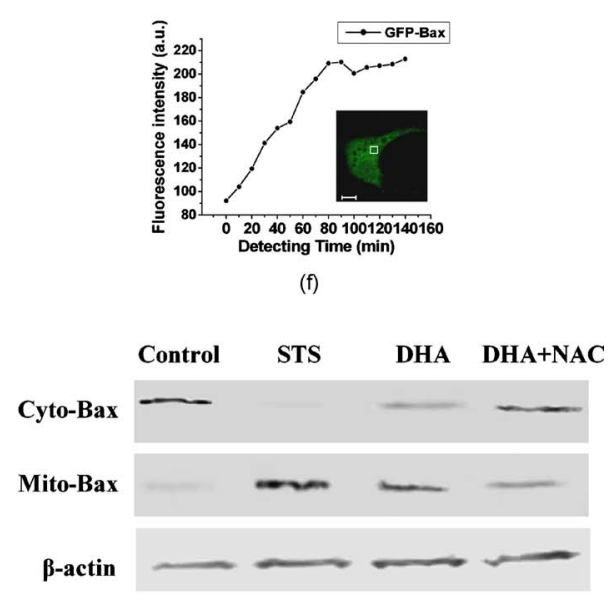

(g)

Fig. 3 DHA-induced ROS-dependent Bax translocation. (a) Typical time-lapse imaging of the mobility of GFP-Bax fusion protein in living cells after photobleaching GFP under different treatments. Upper panel: control cell; middle panel: DHA treatment; lower panel: DHA and NAC cotreatment. Scale bar: $5 \mu \mathrm{m}$. (b) Dynamics of FRAP from 15 to 20 cells in three independent experiments for control, DHA-treated, and DHA and NACcotreated cells. Data were collected at 5-s intervals during recovery. (c) Typical fluorescence images of GFP-Bax distribution in living cells under different treatments. Left panel: DHA treatment alone; right panel: DHA and NAC cotreatment. Scale bar: $20 \mu \mathrm{m}$. (d) Quantification of cells showing GFP-Bax translocation. Cells were treated with $20 \mu \mathrm{g} / \mathrm{ml}$ DHA for 0,24 , and $48 \mathrm{~h}$ in the presence or absence of $5 \mathrm{mM}$ NAC. Results were presented as mean $\pm S D$, collecting from 200 to 300 cells per treatment in 15 to 20 randomly selected image frames from three independent experiments. ${ }^{* *} P<0.01$, compared with control; ${ }^{\# \#} P<0.01$, compared with DHA treatment alone. (e) Time-lapse imaging of Bax translocation into mitochondria inside single living cell. Cells transiently coexpressing GFP-Bax and DsRed-Mito were treated with $20 \mu \mathrm{g} / \mathrm{ml}$ DHA, and GFP-Bax localization at mitochondria was determined based on the overlays of GFP-Bax and DsRed-Mito fluorescence images. Scale bar: $10 \mu \mathrm{m}$. (f) Dynamics of relative fluorescence intensity of GFP-Bax redistribution in the enriched mitochondrial area (indicated by white square) corresponding to Fig. 3(e). (g) Western blot analysis of Bax translocation into mitochondria. Cells were treated with DHA for $48 \mathrm{~h}$ in the presence or absence of NAC, and STS-treated cells were used as a positive control. The cytosolic and mitochondrial proteins were extracted using a mitochondria/cytosol fractionation kit and then analyzed by Western blot using antibodies against Bax and $\beta$-actin. 

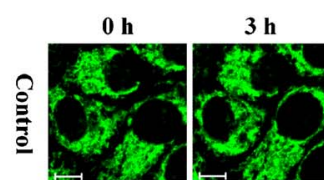

0 h

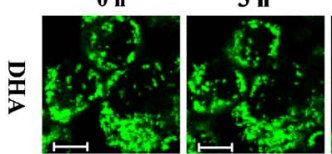

$0 \mathbf{h}$
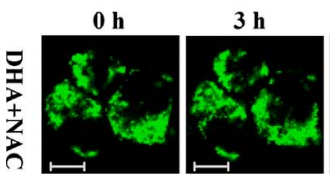

$\longmapsto$

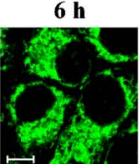

8 h

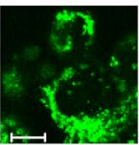

$6 \mathrm{~h}$

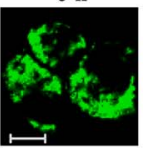

(a)
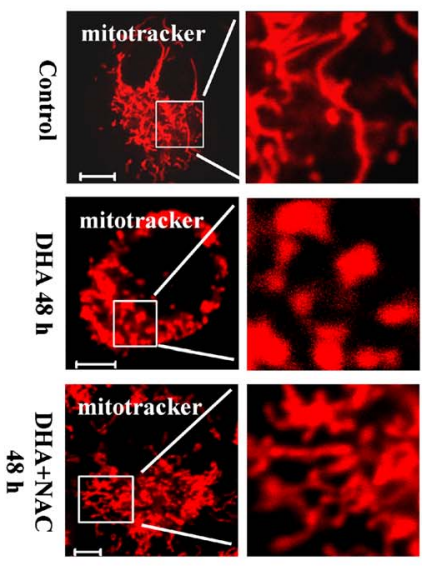

(d)

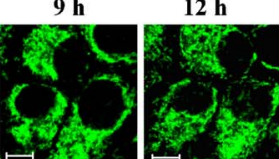

$8.75 \mathrm{~h}$

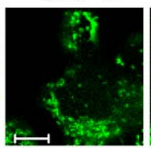

$9 \mathrm{~h}$

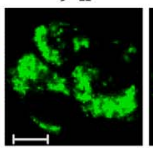

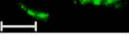
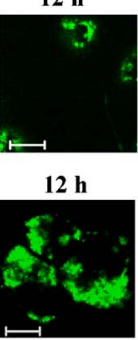

$12 \mathrm{~h}$

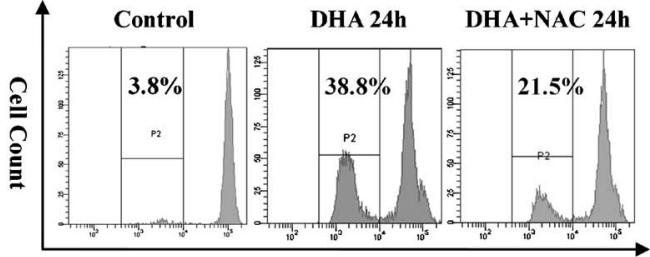

Rhodamine 123 Fluorescence

(c)

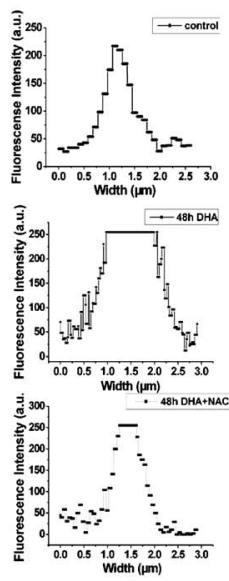

(e)

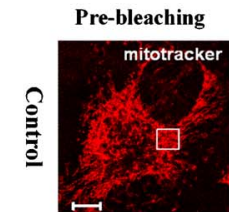

Post-bleaching 0' Post-bleaching 10'
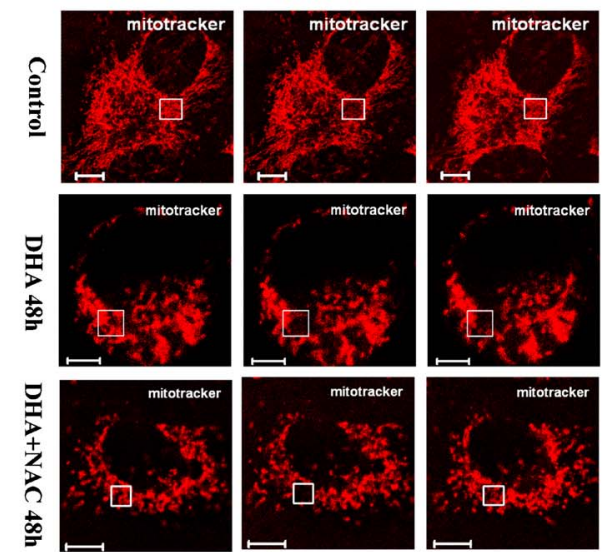

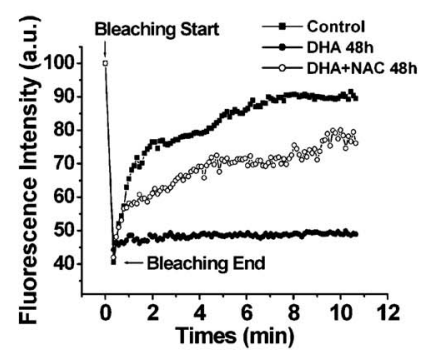

(h)

(g)

Fig. 4 DHA-induced ROS-mediated mitochondrial dysfunction. (a) Time-lapse imaging of cells stained with potential-sensitive dye Rho123. Cells, after treatment with DHA for $24 \mathrm{~h}$ in the presence or absence of NAC, were harvested, and then stained with $5 \mu \mathrm{M}$ Rho123 for 30 min in the dark. Scale bar: $10 \mu \mathrm{m}$. (b) Quantitative analysis of fluorescence intensities of Rho123 in the indicated cells corresponding to Fig. 4(a). (c) FCM analysis of DHA-induced ROS-dependent loss of $\Delta \Psi_{m}$. Cells were cultured with DHA for $24 \mathrm{~h}$ with or without the addition of $5 \mathrm{mM}$ NAC and then stained with $1 \mu \mathrm{M}$ Rho123 before being analyzed by FCM. (d) Typical fluorescence images of mitochondria under different treatments. Upper panel: control cell; middle panel: DHA treatment; lower panel: DHA and NAC cotreatment. Scale bar: $10 \mu \mathrm{m}$. (e) Spatial distribution of mitochondrial size indicated as the white line corresponding to Fig. 4(d). (f) Quantification of mitochondrial width under different treatments. For every condition tested, data for the width were collected from 200 to 300 mitochondria in at least 50 different cells from three independent experiments. ${ }^{* *} P<0.01$, compared with control; ${ }^{\# \#} P<0.01$, compared with DHA treatment alone. (g) Time-lapse imaging of mitochondria inside living cells after photobleaching Mitotracker Red 633 under different treatments. Upper panel: control cell; middle panel: DHA treatment; lower panel: DHA and NAC cotreatment. Images were recorded at 5-s intervals during recovery. Cells, after treatment with DHA for $48 \mathrm{~h}$ in the presence or absence of NAC, were washed three times and then stained by $0.1 \mu \mathrm{M}$ Mitotracker Red 633 for 30 min in the dark. Scale bar: $10 \mu \mathrm{m}$. (h) Quantitative analysis of fluorescence recovery of Mitotracker Red in indicated cells corresponding to Fig. 4(g). (Color online only.) 
upper panel], mitochondria inside DHA-treated cells were punctate and severely swollen [Fig. 4(d), middle panel], which can be significantly attenuated by NAC pretreatment [Fig. 4(d), lower panel]. Figure 4(e) shows the spatial distribution of mitochondrial size, indicated as the white line corresponding to Fig. 4(d). Statistical results of 200 to 300 mitochondria of at least 50 different cells from three independent experiments showed that at $48 \mathrm{~h}$ after DHA treatment, the size of mitochondria increased from $0.29 \pm 0.03 \mu \mathrm{m}$ (control) to $0.68 \pm 0.08 \mu \mathrm{m}$, which reduced to $0.45 \pm 0.11 \mu \mathrm{m}$ in the case of pretreatment with NAC [Fig. 4(f)], surely suggesting that ROS were implicated in DHA-triggered mitochondrial swelling.

Importantly, mitochondrial networks become extensively fragmented into smaller networks during apoptosis. ${ }^{37}$ Rapid recovery of fluorescence after photobleaching is indicative of mitochondrial connectivity, while a failure to recover fluorescence demonstrates mitochondrial discontinuity or fragmentation. Using this method, we found a rapid fluorescence refilling in the long tubular mitochondria after photobleaching Mitotracker Red 633 for control cells [Fig. 4(g), upper panel], while the fluorescence failed to refill for DHA-treated cells [Fig. 4(g), middle panel]. However, fluorescence in the photobleached area recovered significantly for the cells co-treated with DHA and NAC [Fig. 4(g), lower panel], which was consistent with the FRAP curves of quantitative analysis of fluorescence intensities recorded during photobleaching corresponding to Fig. 4(g) [Fig. 4(h)], further demonstrating that DHA-induced mitochondrial fragmentation, associated with the impairment of mitochondrial motility, was also mediated by DHA-elicited ROS.

\subsection{Involvement of $B C l-x L$ in the DHA-Induced Apoptosis and Inhibition of the DHA- Induced Bax Translocation by $B C l-x L$ Overexpression}

It is not clear why mitochondrial networks fragment during apoptosis, but it has been proposed that this may be due to neutralization of the function of one or more members of Bcl-2 family. ${ }^{39}$ Here, we considered whether Bcl-xL, a strongly anti-apoptotic member of the Bcl-2 family, was implicated in the regulation of apoptosis by DHA. As can be seen in Fig. 5(a), pretreatment with HA14-1, a Bcl-xL inhibitor, significantly increased DHA-induced cytotoxicity compared with DHA treatment alone, suggesting that Bcl-xL protected cells against DHA-induced cell death.

To explore whether ROS might mediate the effect of DHA on Bcl-xL, cells were treated with DHA in the presence or absence of NAC, and the corresponding effects on Bcl-xL expression are shown in Fig. 5(b). DHA caused a timedependent decrease in Bcl-xL expression as well as STS treatment, whereas pretreatment of the cells with NAC markedly inhibited the decrease in Bcl-xL expression level [Fig. 5(b)]. These findings implied that ROS were responsible in great part for DHA-induced Bcl-xL down-regulation.

Furthermore, to provide a mechanism insight into how Bcl-xL prevented DHA-induced cell death, we assessed the effect of Bcl-xL overexpression on Bax translocation in DHAtreated cells. For the cells co-expressing CFP-Bax and YFPBcl-xL, time-lapse images showed that after DHA treatment for $30 \mathrm{~h}$, Bax still evenly distributed throughout the cells even in a dead cell (indicated by a white arrow) [Fig. 5(c)], which was quite different from the punctuated distribution of Bax in the DHA-treated cells expressing GFP-Bax alone [Fig. 3], implying that $\mathrm{Bcl}-\mathrm{xL}$ overexpression prevented Bax translocation. The percentage of cells showing Bax translocation for the cells expressing GFP-Bax alone and co-expressing both CFP-Bax and YFP-Bcl-xL at 0 and $24 \mathrm{~h}$ after DHA treatment is shown in Fig. 5(d). DHA treatment induced an increase of Bax translocation from $4.85 \pm 1.5 \%$ (control) to $29 \pm 2.1 \%$, whereas the percentage of cells with DHA-induced Bax translocation markedly decreased to $16.8 \pm 3.0 \%$ in the case of $\mathrm{Bcl}-\mathrm{xL}$ overexpression. These results presented the evidence that Bcl-xL protected cells by preventing DHA-induced Bax translocation, also confirming that Bax activation was of paramount importance in the DHA-provoked apoptotic cell death.

\subsection{Requirement of ROS for DHA-Triggered Cytochrome c Release and Caspase-9 Activation}

The activation of Bax or Bak triggers apoptosis by decreasing the $\Delta \Psi_{m}$, which resulted in the release of cytochrome $c$ from the mitochondrial intermembrane space into the cytosol. ${ }^{22} \mathrm{We}$ first monitored the dynamics of cytochrome $c$ release inside single living cell co-expressing GFP-Cyt. $c$ and DsRed-Mito using a time-lapse confocal fluorescence microscope. Figure 6(a) shows a typical record of real-time images, where the DHA-treated cell was undergoing the release of cytochrome $c$, which became diffusely distributed throughout the cytoplasm. In contrast, pretreatment with NAC markedly postponed cytochrome $c$ release in response to DHA (data not shown). Next, Western blot analysis further confirmed that the release of cytochrome $c$ was significantly inhibited by NAC pretreatment [Fig. 6(b)], implying that ROS were required for cytochrome $c$ release in this apoptotic process.

Due to the fact of cytochrome $c$ release, it was possible to speculate whether the activation of caspase- 9 was involved in DHA-provoked apoptosis, which was detected by FRET acceptor photobleaching analysis and fluorometric assay. First, we used FRET acceptor photobleaching analysis to assess the caspase- 9 activation inside single living cells transiently expressing SCAT9. Our data showed that after photobleaching Venus with a concomitant decrease of fluorescence intensity $\left(\Delta I_{\text {Venus }}\right)$ in the Venus channel, the increase of fluorescence intensity $\left(\Delta I_{C F P}\right)$ in the ECFP channel for DHA-treated cells [Fig. 6(c), right panel] was much less than that for control cells [Fig. 6(c), left panel]. Thus, the ratio of $\Delta I_{C F P} / \Delta I_{\text {Venus }}$ from at least 15 to 20 cells in three independent experiments had a significant decrease at $48 \mathrm{~h}$ after DHA treatment compared with the control [Fig. 6(d)], indicating that DHA induced caspase-9 activation. Furthermore, we also used fluorometric assay to assess the role of ROS in DHA-induced caspase-9 activation. The proteolytic activity that fluorogenic polypeptide Ac-LEHD-AFC can be cleaved by caspase-9-like proteases was associated with caspase-9 activation. STStreated cells were used as a positive control. After incubation with Ac-LEHD-AFC for $2 \mathrm{~h}$, an obvious increase of caspase- 9 activity was observed at $48 \mathrm{~h}$ after DHA treatment compared with control, and the caspase- 9 activation was almost completely attenuated in the presence of NAC pretreatment [Fig. 6(e)], indicating that ROS mediated the DHA- 


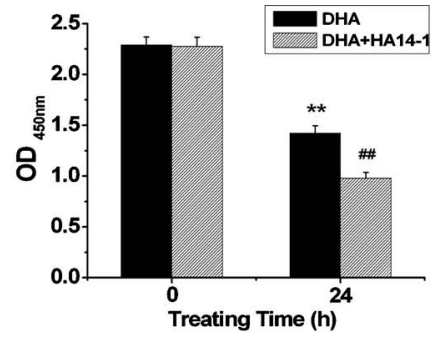

(a)

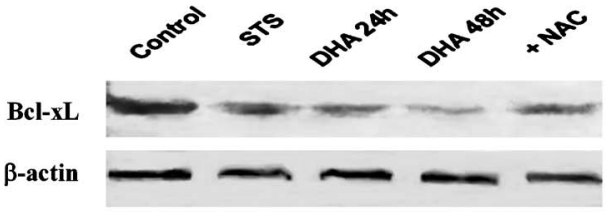

(b)

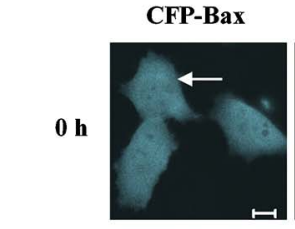

YFP-Bcl-xL
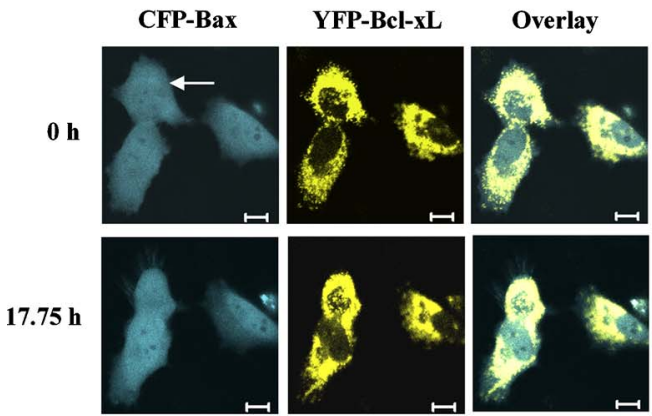

DIC
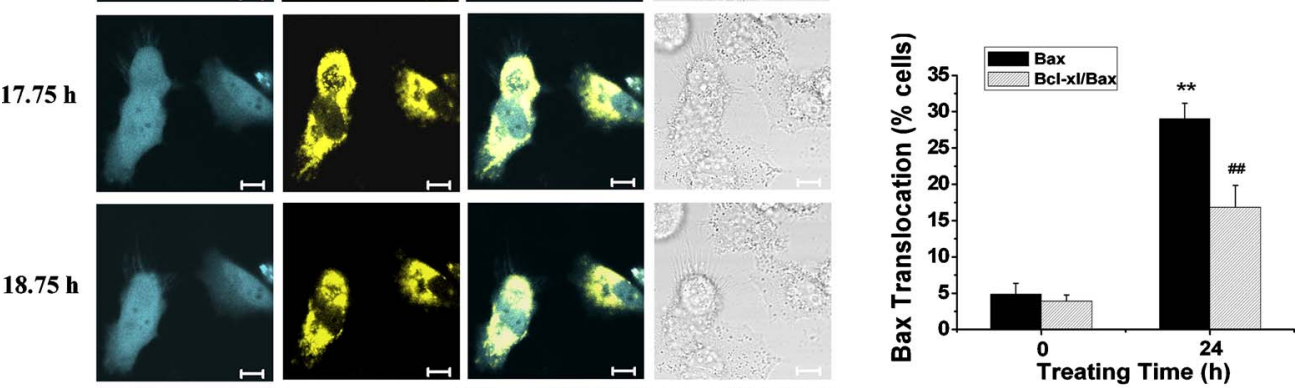

(d)

$30 \mathrm{~h}$
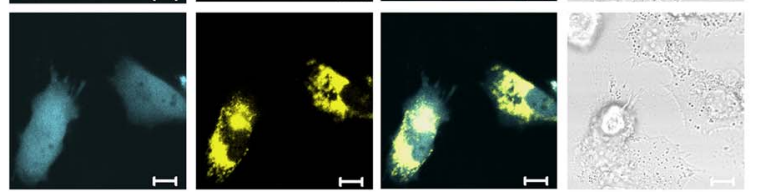

(c)

Fig. 5 Overexpression of $\mathrm{BCl}-\mathrm{xL}$ prevented Bax translocation into mitochondrial membrane. (a) Inhibition of HA14-1, a Bcl-xL inhibitor, on cell viability assessed by CCK-8. Cells were cultured with DHA for 0 or $24 \mathrm{~h}$ with or without the addition of $10 \mu \mathrm{M}$ HA14-1. Experiments were conducted in quadruple wells, and data analyzed with SPSS 10.0 software were expressed as mean \pm SD. ${ }^{* *} P<0.01$, compared with control; ${ }^{\#} P<0.01$, compared with DHA treatment alone. (b) Western blot analysis of the inhibition of DHA on Bcl-xL expression. Cells were treated with DHA for 0,24 , and $48 \mathrm{~h}$, and cotreated with DHA and NAC for $48 \mathrm{~h}$. Bcl-xL expression in control, STS-treated (as a positive control), DHA-treated, and DHA and NAC-cotreated cells were detected by Western blot using antibodies against $\mathrm{Bcl}$-xL and $\beta$-actin. (c) Inhibition of Bcl-xL overexpression on Bax translocation. Cells transiently coexpressing CFP-Bax and YFP-Bcl-xL were treated with $20 \mu \mathrm{g} / \mathrm{ml} \mathrm{DHA}$, and the dynamics of Bax distribution were monitored using time-lapse confocal imaging. Scale bar: $5 \mu \mathrm{m}$. (d) Quantification of cells showing the inhibition of Bcl-xL overexpression on Bax translocation. Cells expressing GFP-Bax or coexpressing CFP-Bax and YFP-Bcl-xL were treated with $20 \mu \mathrm{g} / \mathrm{ml}$ DHA. Results were presented as mean $\pm S D$, collecting in 200 to 300 cells per treatment in 15 to 20 randomly selected image frames from three independent experiments. ${ }^{* *} P<0.01$, compared with GFP-Bax-transfected control; ${ }^{\# \#} P<0.01$, compared with GFP-Bax-transfected cells after DHA treatment.

induced caspase-9 activation. Collectively, these results demonstrated that ROS acted upstream of DHA-induced cytochrome $c$ release and caspase- 9 activation.

\subsection{DHA-Induced ROS-Dependent Caspase-3 Activation}

It is reported that the activated caspase- 9 then cleaves and activates other downstream caspases such as caspase- $3,{ }^{40}$ and we recently found that DHA induced caspase-3-dependent apoptosis in ASTC-a-1 cells. ${ }^{3}$ In order to verify whether the DHA-induced caspase-3 activation was mediated by ROS generation, we used fluorometric assay, fluorescence emission spectra, and Western blot analysis to assess the effect of NAC on the DHA-induced caspase-3 activation. STS-treated cells were used as a positive control. For fluorometric assay, activated caspase-3, but not pro-caspase-3, does exert proteolytic activation on the Ac-DEVD-AFC substrate. As shown in Fig. 7(a), compared with the control, DHA induced a nearly twofold increase of caspase- 3 activity that could be almost completely blocked by NAC pretreatment, implying that ROS mediated the DHA-induced caspase-3 activation. Emission spectral analysis in living cells stably expressing SCAT3 showed that bimodal emission peaks of SCAT3 were observed around $481 \mathrm{~nm}$ and $524 \mathrm{~nm}$ in control cells, but in DHAtreated cells, the increase at 481-nm and the decrease at 524-nm emission peak were indicative of caspase-3 activation [Fig. 7(b)]. However, NAC pretreatment significantly attenuated the DHA-induced decrease at the 524-nm peak [Fig. 7(b)], demonstrating that ROS played a critical role in DHAtriggered caspase- 3 activation. Moreover, Western blot analysis further confirmed these findings that NAC effectively blocked the DHA-induced pro-caspase-3 cleavage [Fig. 7(c)]. 


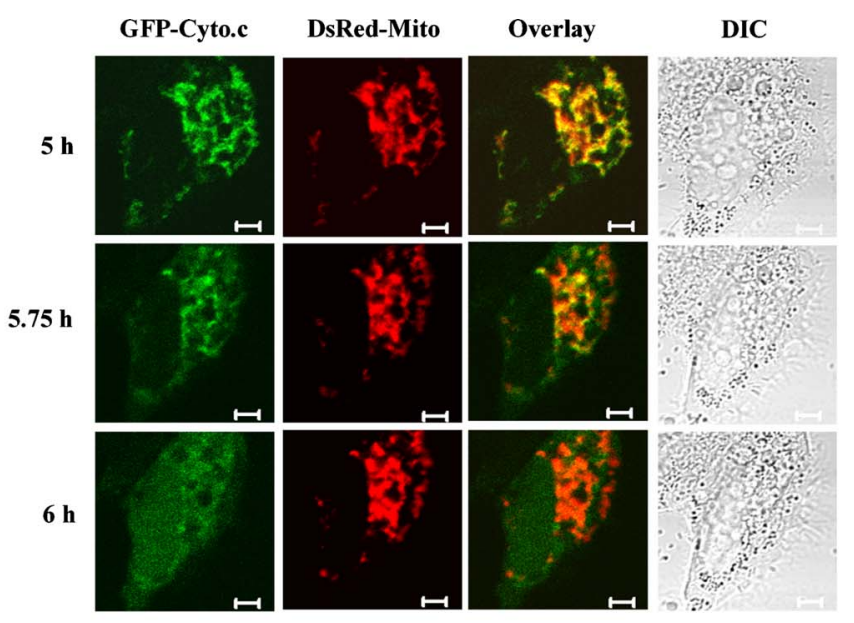

(a)

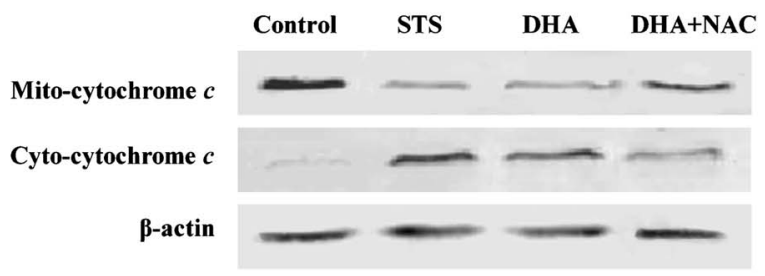

(b)
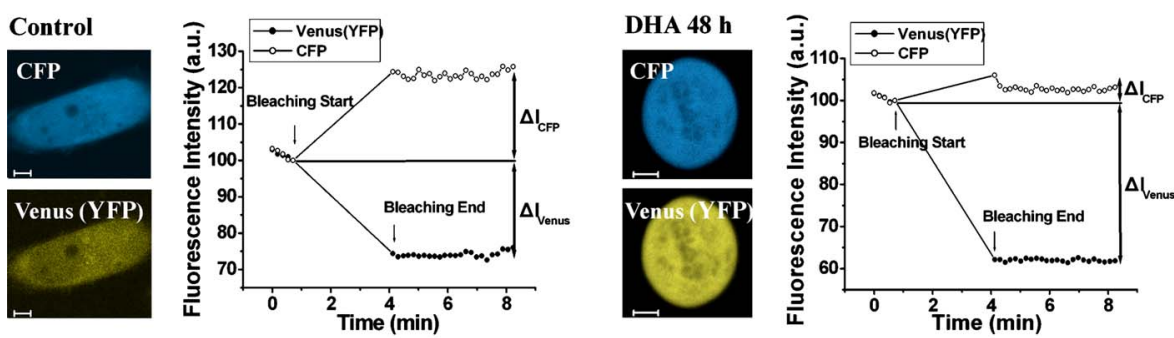

(c)
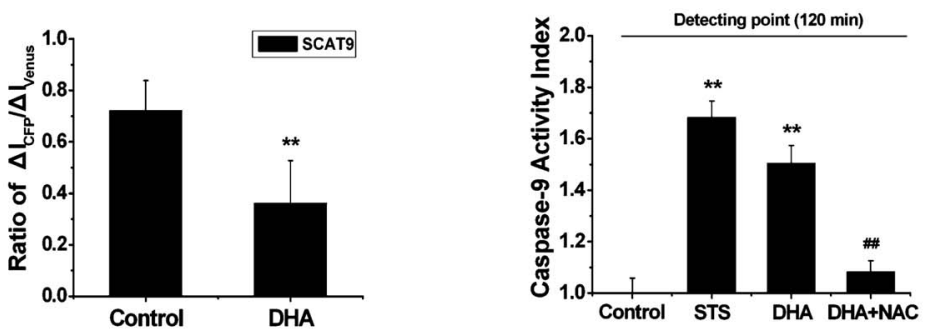

(d)

(e)

Fig. 6 DHA-induced ROS-dependent cytochrome $c$ release and caspase-9 activation. (a) Typical time-lapse imaging of cytochrome $c$ release inside a single living cell. Cells transiently coexpressing GFP-Cyt. $c$ and DsRed-Mito were treated with $20 \mu \mathrm{g} / \mathrm{ml}$ DHA, and the release of GFP-Cyt. $c$ from mitochondria to cytosol was determined based on the overlays of GFP-Cyt. $c$ and DsRed-Mito fluorescence images. Scale bar: $5 \mu \mathrm{m}$. (b) Western blot analysis of the release of cytochrome $c$ from mitochondria to cytosol. Cells were treated with DHA for $48 \mathrm{~h}$ in the presence or absence of NAC, and STS-treated cells were used as a positive control. The cytosolic and mitochondrial proteins were extracted using a mitochondria/cytosol fractionation kit and then analyzed by Western blot using antibodies against cytochrome $c$ and $\beta$-actin. (c) Typical fluorescence images and the corresponding dynamics of fluorescence intensity of both ECFP and Venus channels for control cell (left panel) and DHA-treated cell (right panel) transiently expressing with SCAT9 before and after photobleaching Venus. The photobleaching laser is $514 \mathrm{~nm}$, and the FRET excitation laser is $458 \mathrm{~nm} . \Delta I_{\text {CFP }}$ is the increase of ECFP intensity, and $\Delta I_{\text {Venus }}$ is the decrease of Venus intensity. All initial intensity is normalized to 100. Scale bar: $5 \mu \mathrm{m}$. (d) Quantitative analysis of the ratio of $\Delta I_{C F P} / \Delta I_{V e n u s}$ as described in Sec. 2. Data were obtained from 15 to 20 cells in three independent experiments. ${ }^{* *} P<0.01$, compared with control. (e) Detection of caspase-9 activation by fluorometric assay. Cells were treated with DHA for $48 \mathrm{~h}$ in the presence or absence of NAC, and STS-treated cells were used as a positive control. Caspase-9 activity was measured using the fluorescence substrate Ac-LFHD-AFC, as described in Sec. 2, and the activation index was determined as the ratio between the activity in extracts of treated cells and that measured in extracts of control cells. Data were analyzed with SPSS 10.0 software from three independent experiments. ${ }^{* *} P<0.01$, compared with control; ${ }^{\# \#} P<0.01$, compared with DHA-treated cells. 


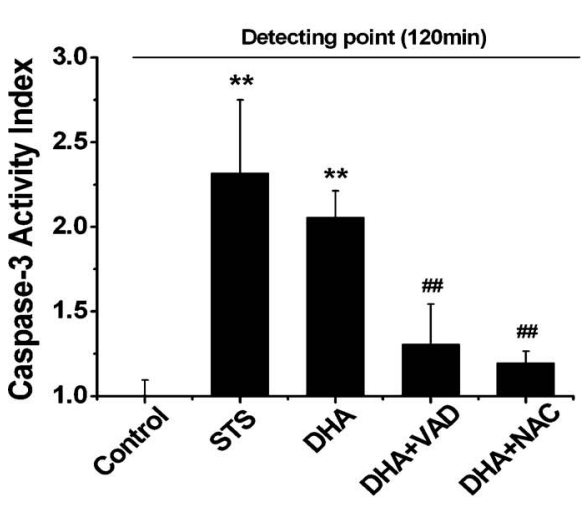

(a)

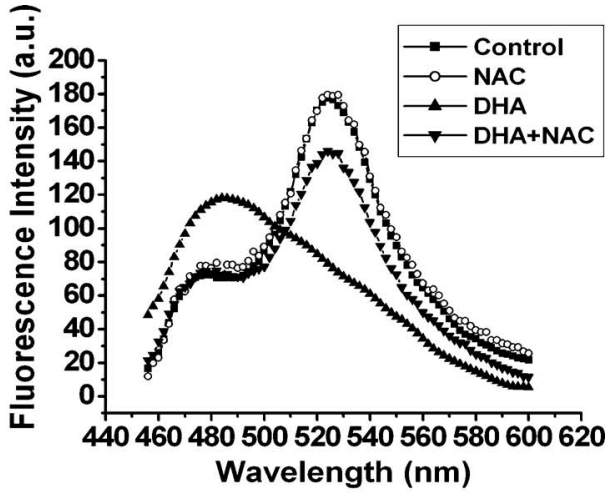

(b)

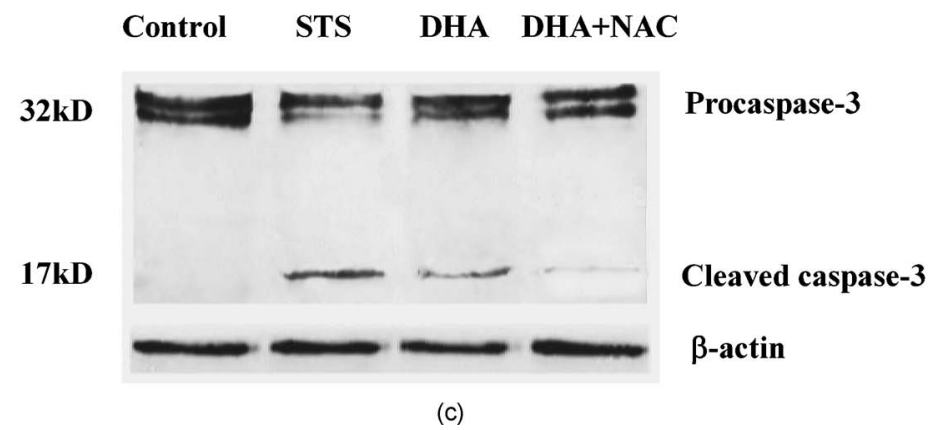

Fig. 7 DHA-induced ROS-dependent caspase-3 activation. (a) Detection of caspase-3 activation by fluorometric assay. Cells were treated with DHA for $48 \mathrm{~h}$ in the presence or absence of NAC, and STS-treated cells were used as a positive control. Caspase- 3 activity was measured using the fluorescence substrate Ac-DEVD-AFC, as described in Sec. 2, and the activation index was determined as the ratio between the activity in extracts of treated cells and that measured in extracts of control cells. Data were analyzed with SPSS 10.0 software from three independent experiments. ${ }^{* *} P<0.01$, compared with control; ${ }^{\# \#} P<0.01$, compared with DHA-treated cells. (b) Emission spectral analysis of caspase-3 activation in living cells. Cells stably expressing SCAT3 were treated with $20 \mu \mathrm{g} / \mathrm{ml} \mathrm{DHA} \mathrm{for} 0$ or $48 \mathrm{~h}$ with or without the addition of NAC. (c) Western blot analysis of DHA-induced ROS-mediated caspase-3 activation. Cells were treated with DHA for $48 \mathrm{~h}$ in the presence or absence of NAC, and STS-treated cells were used as a positive control. Protein levels of caspase- 3 in control, STS-treated, DHA-treated, and DHA and NAC-cotreated cells were examined by Western blot using antibodies against caspase- 3 and $\beta$-actin.

Our data suggested that DHA-triggered caspase-3 activation was ROS-dependent.

\subsection{DHA-Triggered ROS-Dependent Caspase-8 and Bid Activation}

In addition to the mitochondrial death (intrinsic) pathway activated caspase- 8 amplifies the apoptotic signal in the extrinsic pathway, either by directly activating the downstream caspase- 3 or by cleaving Bid to form t-Bid. ${ }^{32,41}$ Hence, we next explored whether caspase-8 and Bid activation were involved in DHA-triggered apoptosis, in which DHA-elicited ROS acted as an upstream modulator. First, the activation of caspase- 8 was evaluated by determining fluorogenic AFC release. Ac-IETD-AFC, which can be cleaved by caspase-8-like proteases, was associated with caspase- 8 activation. STStreated cells were used as a positive control. As shown in Fig. 8(a), compared with the control, DHA induced a nearly 1.8fold increase of caspase- 8 activity, which was significantly but not completely suppressed by the NAC pretreatment, indicating that ROS partially mediated the DHA-triggered caspase- 8 activation. Second, we used FRET acceptor photobleaching analysis to assess the effect of DHA on Bid cleavage in single living cells transiently expressing YFP-Bid-CFP
(FRET-Bid). Our data showed that after photobleaching YFP with a concomitant decrease of fluorescence intensity $\left(\Delta I_{Y F P}\right)$ in the YFP channel, the increase of fluorescence intensity $\left(\Delta I_{C F P}\right)$ in the CFP channel for DHA-treated cells [Fig. 8(b), lower panel] was much less than that for control cells [Fig. 8 (b), upper panel]. Thus, the ratio of $\Delta I_{C F P} / \Delta I_{Y F P}$ from at least 15 to 20 cells in three independent experiments had a significant decrease at $48 \mathrm{~h}$ after DHA treatment compared with the control [Fig. 8(c)], suggesting that DHA induced Bid cleavage, which was probably due to DHA-triggered caspase- 8 activation. Third, the dependence of tBid translocation on ROS generation was next investigated in single living cells co-expressing CFP-Bid and DsRed-Mito. Figure 8(d) shows the typical images of Bid distribution for control cells and DHA-treated cells. CFP-Bid had a diffuse and cytoplasmic localization in control cells, while DHA-treated cells showed Bid translocation from cytoplasm to mitochondria, which was remarkably blocked by NAC pretreatment (data not shown), as revealed by the overlaps of CFP-Bid and DsRed-Mito fluorescence images. Statistical results of the percentage of cells showing Bid translocation in at least 150 to 200 cells in three independent experiments are shown in Fig. 8(e), also demonstrating that DHA induced time- 

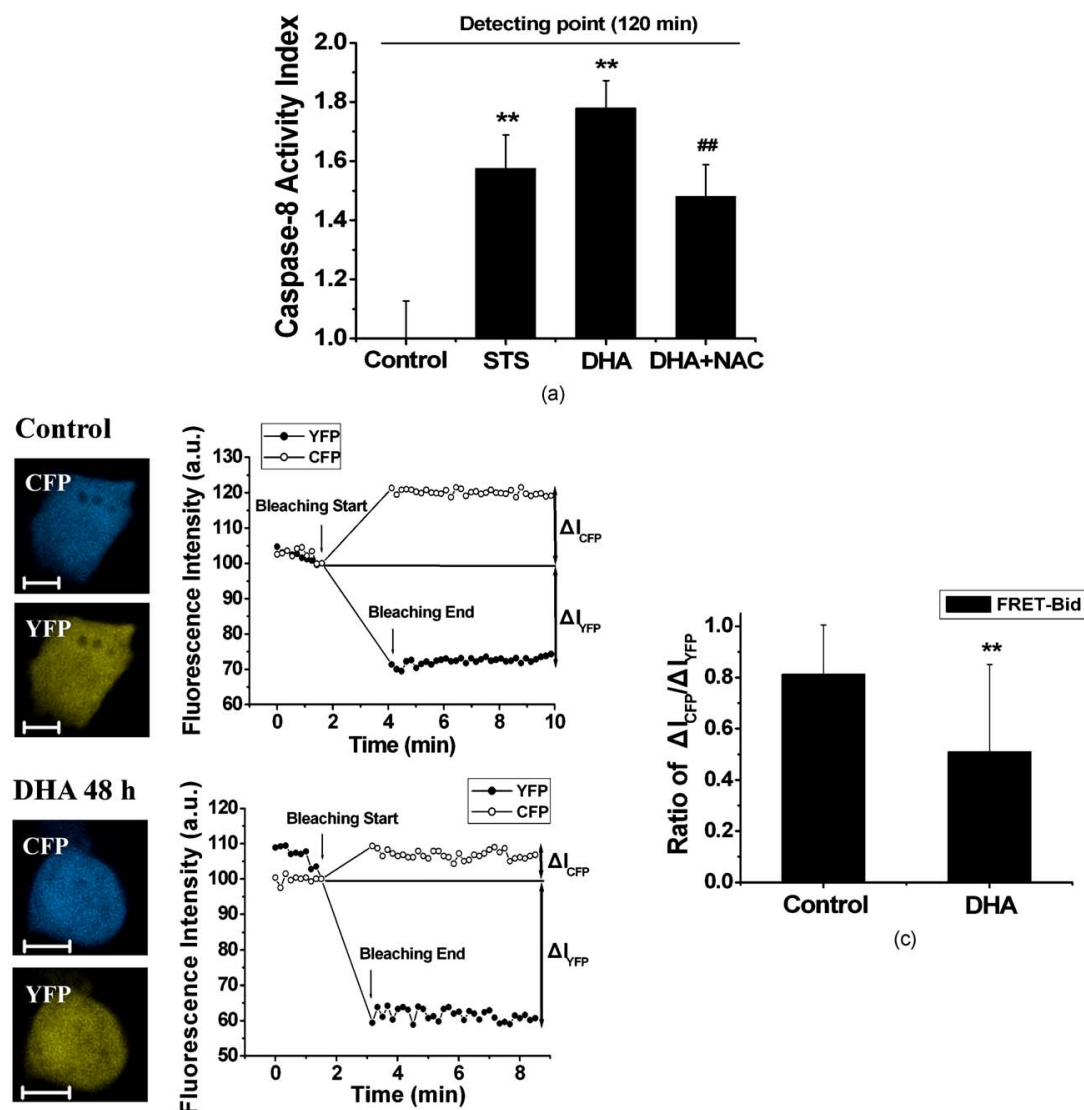

(b)
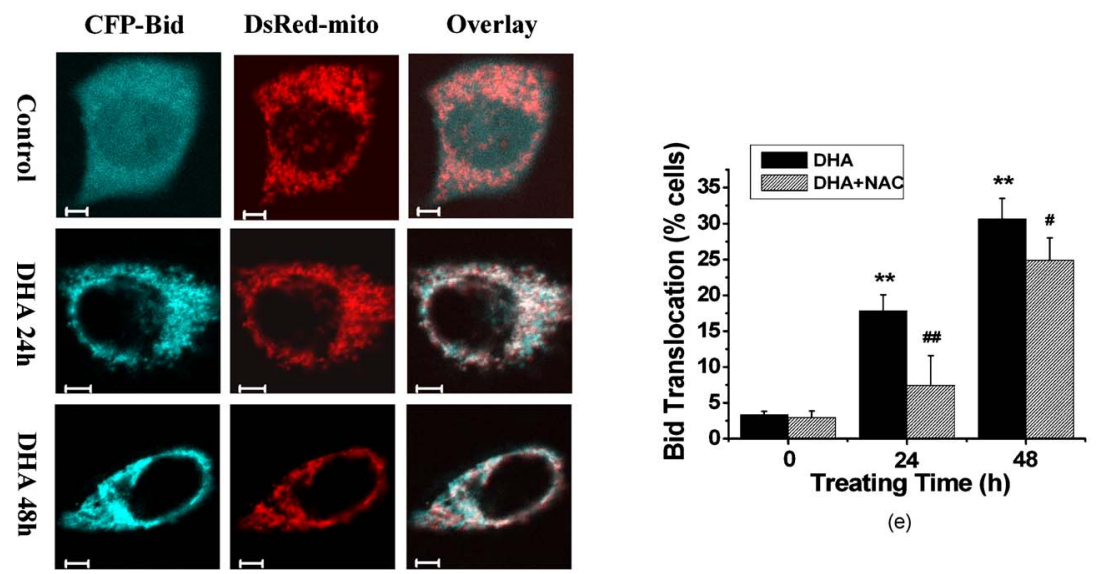

(d)

Fig. 8 DHA-triggered ROS-dependent caspase- 8 and Bid activation. (a) Detection of caspase- 8 activation by fluorometric assay. Cells were treated with DHA for $48 \mathrm{~h}$ in the presence or absence of NAC, and STS-treated cells were used as a positive control. Caspase-8 activity was measured using the fluorescence substrate Ac-IETD-AFC, as described in Sec. 2, and the activation index was determined as the ratio between the activity in extracts of treated cells and that measured in extracts of control cells. Data were analyzed with SPSS 10.0 software from three independent experiments. ${ }^{* *} P<0.01$, compared with control; ${ }^{\# \#} P<0.01$, compared with DHA-treated cells. (b) Typical fluorescence images and the corresponding dynamics of fluorescence intensity of both CFP and YFP channels for control cell (upper panel) and DHA-treated cell (lower panel) transiently expressing FRET-Bid before and after photobleaching YFP. The photobleaching laser is $514 \mathrm{~nm}$, and the FRET excitation laser is $458 \mathrm{~nm}$. $\Delta I_{C F P}$ is the increase of CFP intensity, and $\Delta I_{\text {YFP }}$ is the decrease of YFP intensity. All initial intensity is normalized to 100 . Scale bar: $10 \mu \mathrm{m}$. (c) Quantitative analysis of the ratio of $\Delta I_{C F P} / \Delta I_{Y F P}$, as described in Sec. 2. Data were obtained from 15 to 20 cells in three independent experiments. ${ }^{* *} P<0.01$, compared with control. (d) Representative images of Bid translocation into mitochondria. Cells transiently coexpressing CFP-Bid and DsRed-Mito were treated with $20 \mu \mathrm{g} / \mathrm{ml} \mathrm{DHA}$ for 0,24 , and $48 \mathrm{~h}$, and the translocation of Bid from cytoplasm to mitochondria was determined based on the overlays of CFP-Bid and DsRed-Mito fluorescence images. Scale bar: $5 \mu \mathrm{m}$. (e) Quantification of cells showing CFP-Bid translocation into mitochondria. Cells were treated with $20 \mu \mathrm{g} / \mathrm{ml} \mathrm{DHA}$ for 0,24 , and $48 \mathrm{~h}$ in the presence or absence of $5 \mathrm{mM}$ NAC. Results were presented as mean $\pm \mathrm{SD}$, collecting in 150 to 200 cells per treatment in 15 to 20 randomly selected image frames from three independent experiments. ${ }^{* *} P<0.01$, compared with control; ${ }^{\#} P<0.05,{ }^{\#} P<0.01$, compared with DHA treatment alone. 
dependent Bid translocation to mitochondria, which was effectively attenuated by NAC pretreatment.

Taken together, these results consistently indicated that DHA-elicited ROS acted upstream of the caspase- 8 and Bid activation.

\section{Discussion}

Although continued efforts have been made to identify novel biological and molecular targets for cancer therapeutics, lung adenocarcinoma is still considered as a largely incurable disease. ${ }^{42}$ As such, this present study focuses on investigating the novel molecular mechanism by which DHA induced apoptotic cell death using fluorescence imaging techniques, which might help discover a promising new target for DHA to exert its anticancer activity. We provide the first and direct evidence that DHA induces apoptosis in ASTC-a-1 cells via the ROS-dependent mitochondrial and death receptor pathway, which may serve as important clinical significance to guide the treatment of lung cancer.

It is widely accepted that mitochondria are both a major source of ROS and a target for their damaging effects due to ROS. $^{43}$ Some reports showed that ROS generation during mitochondria-dependent apoptosis occurs downstream of the release of mitochondrial proapoptotic factors to cytosol. ${ }^{44}$ In many apoptosis models, however, ROS act as upstream signaling molecules that initiate cell death. ${ }^{14}$ In our experimental system, a burst of exogenous ROS generation is mostly due to the reaction of an endoperoxide bridge of DHA with heme irons, ${ }^{24}$ which is verified by our observations that DHAinduced ROS bursting occurs throughout the whole cells, not just from mitochondria [Fig. 1(a)]. Thus, it appears to be reasonable that a large amount of ROS elicited from DHA cause damage to subcellular organelles, especially mitochondria. Specifically, we next question whether the DHA-elicited ROS act upstream in the cell death signaling pathway or whether it is accumulated later as a result of mitochondrial membrane permeabilization. Our data that NAC prevents DHA-induced cell death upstream of $\Delta \Psi_{m}$ collapse and cytochrome $c$ release supports the notion that DHA-elicited ROS lie upstream of the mitochondrial dysfunctions.

In this study, fluorescence recovery after photobleaching (FRAP) was used for the first time to measure the mobility of GFP-Bax and the integrality of mitochondria in DHA-induced apoptosis inside single living cells. In response to mitochondrial-dependent stimuli, except for Bax, Bim and Bad also become activated and translocated to the mitochondrial outer membrane. ${ }^{22,45,46}$ We here further demonstrate that DHA-elicited ROS act upstream of Bax activation in the mitochondrial apoptotic signaling pathway (Fig. 3) and that overexpression of Bcl-xL protects against DHA-induced Bax activation at the mitochondrial membrane and subsequent cell death (Fig. 5). Moreover, since the roles of Bim and Bad in DHA-induced apoptosis have not been established, we found that Bim aggregated and translocated to ER rather than mitochondria, while Bad was not activated after exposure to DHA compared with STS treatment (data not shown). Collectively, these findings implicate the importance of the Bcl-2 family proteins as vital regulators that DHA triggers during this apoptotic process, in which DHA-elicited ROS play a key role.
Our present findings demonstrate that ROS act upstream of Bax activation in the cell death pathway triggered by DHA. However, we do not address the mechanisms by which ROS cause Bax activation. It has been reported that mitogen activated protein kinase (MAPK) pathways are activated in response to changes of the extracellular or intracellular environment and have been shown to be important in Bax activation in response to oxidant stress. ${ }^{47}$ Our observations that the MAPK pathway failed to be activated in DHA-induced apoptosis (data not shown) strongly indicated the existence of other links between DHA-elicited ROS and Bax activation. In addition, we found that DHA treatment induced a direct BclxL-Bax interaction, which lasted for about $40 \mathrm{~min}$ from the initiation of Bax redistribution to the formation of Bax clusters (data not shown). In this model, Bcl-xL is considered to delay or prevent the DHA-induced Bax translocation, and the accumulated Bax on mitochondria may antagonize the inhibitory effects of Bcl-xL, subsequently triggering mitochondrial membrane permeabilization. Another interesting event presented in this work is the inhibition of NAC pretreatment on the DHA-induced down-regulation of $\mathrm{Bcl}-\mathrm{xL}$ expression level, demonstrating that DHA-elicited ROS down-regulate Bcl-xL expression, and then promote Bax translocation and activation and release of cytochrome $c$, which initiates the activation of caspase- 9 and caspase-3. Therefore, it is tempting to speculate here that $\mathrm{Bcl}-\mathrm{xL}$ acts as a possible mediator between ROS and Bax activation.

In addition to the mitochondrial death (intrinsic) pathway, another cell death pathway is the extrinsic cell death pathway, in which caspase- 8 plays an important role. ${ }^{32}$ In this report, the FRET acceptor photobleaching technique was conducted to monitor the DHA-induced ROS-dependent caspase- 8 and Bid activation, suggesting that ROS are also a critical regulator of the death receptor pathway (Fig. 8). Currently, caspase- 8 is shown to be involved not only in the death receptor pathway, but also in the mitochondrial apoptosis pathway by cleaving Bid to form truncated-Bid, which is known to activate Bax and trigger Bax-driven mitochondrial membrane permeabilization. ${ }^{32,41}$ Zhang and Bowden proposed that $\mathrm{Bcl}-\mathrm{xL}$ also binds to tBid, preventing its interaction with Bax/ Bak and their translocation to mitochondria. ${ }^{48}$ It is possible that DHA-elicited ROS down-regulate the expression of Bcl$\mathrm{xL}$, which may reduce the interaction of Bcl-xL-tBid so as to promote Bax translocation and activation, suggesting that DHA-elicited ROS mediate the caspase- 8 activation, Bid cleavage, and tBid translocation, which may also participate in the DHA-triggered mitochondrial pathway.

In this report, NAC only partially protected cells from DHA-induced apoptosis [Fig. 1(d) and Fig. 2(d)], indicating the co-existence of ROS-dependent and ROS-independent pathways in DHA-induced apoptosis. Specifically, the ROSdependent pathway involved DHA-induced Bax translocation (Fig. 3), mitochondrial dysfunction (Fig. 4), and cytochrome $c$ release [Fig. 6(b)], which could not be completely inhibited by NAC co-treatment; however, DHA-induced caspase- 9 and caspase- 3 activation could be almost rescued by NAC cotreatment [Fig. 6(e) and Fig. 7]. These results imply that ROS is indispensable for DHA-induced caspase-9/caspase-3dependent pathway, while there have been ROS-independent pathways that also regulate DHA-induced Bax translocation, 
mitochondrial dysfunction, and cytochrome $c$ release, etc. Additionally, DHA-induced caspase- 8 and Bid activation were not fully relied on DHA-elicited ROS [Figs. 8(a) and 8(e)], indicating that ROS play only a small role in the DHAactivated extrinsic pathway.

Collectively, our findings lead to the conclusion that as a first-line antimalarial drug, DHA induces ROS-mediated apoptotic cell death via both the death receptor and mitochondrial pathway in ASTC-a-1 cells, which gives a better understanding of DHA's actions and mechanisms and provides evidence for the rational application of DHA as a novel potential anticancer drug in the clinical treatment of lung adenocarcinoma.

\section{Acknowledgments}

We thank Prof. M. Miura for providing us with SCAT9 and SCAT3 plasmids, Prof. K. Taira for providing FRET-Bid and CFP-Bid plasmids, Prof. Y. Gotoh for providing DsRed-Mito plasmid, Dr. G. J. Gores for providing the GFP-Cyt.c plasmid, and Prof. R. J. Youle for providing GFP-Bax plasmid. This study was supported by the Program for Changjiang Scholars and Innovative Research Team in University (Grant No. IRT0829), the National Natural Science Foundation of China (Grant No. 30670507), and the Natural Science Foundation of Guangdong Province (Grant Nos. 8151063101000031 and 9251063101000009$)$.

\section{References}

1. R. K. Haynes, "Artemisinin and derivatives: the future for malaria treatment," Curr. Opin. Infect. Dis. 14(6), 719-726 (2001)

2. N. P. Singh and H. C. Lai, "Artemisinin induces apoptosis in human cancer cells," Anticancer Res. 24(4), 2277-2280 (2004).

3. Y. Y. Lu, T. S. Chen, J. L. Qu, W. L. Pan, L. Sun, and X. B. Wei, "Dihydroartemisinin (DHA) induces caspase-3-dependent apoptosis in human lung adenocarcinoma ASTC-a-1 cells," J. Biomed. Sci. 16, 16 (2009).

4. S. W. Fesik, "Promoting apoptosis as a strategy for cancer drug discovery," Nat. Rev. Cancer 5(11), 876-885 (2005).

5. J. Hou, D. Wang, R. Zhang, and H. Wang, "Experimental therapy of hepatoma with artemisinin and its derivatives: in vitro and in vivo activity, chemosensitization, and mechanisms of action," Clin. Cancer Res. 14(17), 5519-5530 (2008).

6. T. Chen, M. Li, R. W. Zhang, and H. Wang, "Dihydroartemisinin induces apoptosis and sensitizes human ovarian cancer cells to carboplatin therapy," J. Cell Mol. Med. 13(7), 1358-1370 (2009).

7. G. L. Disbrow, A. C. Baege, K. A. Kierpiec, H. Yuan, J. A. Centeno, C. A. Thibodeaux, D. Hartmann, and R. Schlegel, "Dihydroartemisinin is cytotoxic to papillomavirus-expressing epithelial cells in vitro and in vivo," Cancer Res. 65(23), 10854-10861 (2005).

8. A. E. Mercer, J. L. Maggs, X. M. Sun, G. M. Cohen, J. Chadwick, P. M. O'Neill, and B. K. Park, "Evidence for the involvement of carbon-centered radical in the induction of apoptosis cell death by artemisinin compounds," J. Biol. Chem. 282(13), 9372-9382 (2007)

9. J. J. Lu, L. H. Meng, Y. J. Cai, Q. Chen, L. J. Tong, L. P. Lin, and J. Ding, "Dihydroartemisinin induces apoptosis in HL-60 leukemia cells dependent of iron and p38 mitogen-activated protein kinase activation but independent of reactive oxygen species," Cancer Biol. Ther. 7(7), 1017-1023 (2008).

10. J. Lee, H. J. Zhou, and X. H. Wu, "Dihydroartemisinin downregulates vascular endothelial growth factor (VEGF) expression and induces apoptosis in chronic myeloid leukemia K562 cells," Cancer Chemother. Pharmacol. 57(2), 213-220 (2006).

11. Y. J. Suzuki, H. J. Forman, and A. Sevanian, "Oxidants as stimulators of signal transduction," Free Radic Biol. Med. 22(1-2), 269-285 (1997).

12. M. Benhar, D. Engelberg, and A. Levitzki, "ROS, stress-activated kinases, and stress signaling in cancer," EMBO Rep. 3(5), 420-425 (2002).
13. E. Gottlieb, M. G. Vander Heiden, and C. B. Thompson, "Bcl-XL prevents the initial decrease in mitochondrial membrane potential and subsequent reactive oxygen species production during tumor necrosis factor alpha-induced apoptosis," Mol. Cell. Biol. 20(15), 5680-5689 (2000).

14. L. J. Buccellato, M. Tso, O. I. Akinci, N. S. Chandel, and G. R. Budinger, "Reactive oxygen species are required for hyperoxiainduced Bax activation and cell death in alveolar epithelial cells," $J$. Biol. Chem. 279(8), 6753-6760 (2004).

15. S. Shukla and S. Gupta, "Apigenin-induced prostate cancer cell death is initiated by reactive oxygen species and p53 activation," Free Radic Biol. Med. 44(10), 1833-1845 (2008).

16. Å. B. Gustafsson and R. A. Gottlieb, "Bcl-2 family members and apoptosis, taken to heart," Am. J. Physiol.: Cell Physiol. 292(1), C45-C51 (2007).

17. S. Cory, D. C. S. Huang, and J. M. Adams, "The Bcl-2 family: roles in cell survival and oncogenesis," Oncogene 22(53), 8590-8607 (2003).

18. K. Wakabayashi, H. Saito, H. Ebinuma, Y. Saito, T. Takagi, M. Nakamura, A. Umezawa, J. Hata, and H. Ishii, "Bcl-2 related proteins are dramatically induced at the early stage of differentiation in human liver cancer cells by a histone deacetylase inhibitor projecting an antiapoptotic role during this period," Oncol. Rep. 7(2), 285-288 (2000).

19. X. X. Cao, I. Mohuiddin, F. Ece, D. J. McConkey, and W. R. Smythe, "Histone deacetylase inhibitor downregulation of bcl-xl gene expression leads to apoptotic cell death in mesothelioma," Am. J. Respir Cell Mol. Biol. 25(5), 562-568 (2001).

20. J. E. Chipuk, M. Bhat, A. Y. Hsing, J. Ma, and D. Danielpour, "Bcl-xL blocks transforming growth factor- $\beta 1$-induced apoptosis by inhibiting cytochrome $c$ release and not by directly antagonizing Apaf-1-dependent caspase activation in prostate epithelial cells," $J$. Biol. Chem. 276(28), 26614-26621 (2001).

21. J. M. Adams and S. Cory, "Life-or-death decisions by the Bcl-2 protein family," Trends Biochem. Sci. 26(1), 61-66 (2001).

22. A. Nechushtan, C. L. Smith, I. Lamensdorf, S. H. Yoon, and R. J. Youle, "Bax and Bak coalesce into novel mitochondria-associated clusters during apoptosis," J. Cell Biol. 153(6), 1265-1276 (2001).

23. J. Y. Byun, M. J. Kim, D. Y. Eum, C. H. Yoon, W. D. Seo, K. H. Park, J. W. Hyun, Y. S. Lee, J. S. Lee, M. Y. Yoon, and S. J. Lee, "Reactive oxygen species-dependent activation of Bax and PARP-1 is required for mitochondrial cell death induced by triterpenoid pristimerin in human cervical cancer cells," Mol. Pharmacol. 76(4), 734744 (2009)

24. P. C. Li, E. Lam, W. P. Roos, M. Z. Zdzienicka, B. Kaina, and T. Efferth, "Artesunate derived from traditional Chinese medicine induces DNA damage and repair," Cancer Res. 68(11), 4347-4351 (2008).

25. J. T. Zhang, D. Xing, and X. J. Gao, "Low-power laser irradiation activates Src tyrosine kinase through reactive oxygen speciesmediated signaling pathway," J. Cell Physiol. 217(2), 518-528 (2008).

26. J. Q. Lin, Z. H. Zhang, J. Yang, S. Q. Zeng, B. F. Liu, and Q. M. Luo, "Real-time detection of caspase-2 activation in a single living HeLa cell during cisplatin-induced apoptosis," J. Biomed. Opt. 11(2), 024011-024016 (2006)

27. S. N. Wu, D. Xing, F. Wang, T. S. Chen, and W. R. Chen, "Mechanistic study of apoptosis induced by high fluence low power laser irradiation using fluorescence imaging techniques," J. Biomed. Opt. 12(6), 064015 (2007).

28. J. Yang, Z. H. Zhang, J. Lin, L. Lu, B. F. Liu, S. Q. Zeng, and Q. M. Luo, "Detection of MMP activity in living cells by a genetically encoded surface-displayed FRET sensor," BBA-Mol. Cell Res. 1773(3), 400-407 (2007).

29. Y. X. Wu, D. Xing, and W. R. Chen, "Single cell FRET imaging for determination of pathway of tumor cell apoptosis induced by photofrin-PDT," Cell Cycle 5(7), 729-734 (2006).

30. J. Braga, J. M. P. Desterro, and M. Carmo-Fonseca, "Intracellular macromolecular mobility measured by fluorescence recovery after photobleaching with confocal laser scanning microscopes," Mol. Biol. Cell 15(10), 4749-4760 (2004).

31. K. Takemoto, T. Nagai, A. Miyawaki, and M. Miura, "Spatiotemporal activation of caspase revealed by indicator that is insensitive to environmental effects," J. Cell Biol. 160(2), 235-243 (2003). 
32. R. Onuki, A. Nagasaki, H. Kawasaki, T. Baba, T. Q. P. Uyeda, and K. Taira, "Confirmation by FRET in individual living cells of the absence of significant amyloid $\beta$-mediated caspase- 8 activation," Proc. Natl. Acad. Sci. U.S.A. 99(23), 14716-14721 (2002).

33. G. Santamaria, M. Martinez-Diez, I. Fabregat, and C. M. Cuezva, "Efficient execution of cell death in non-glycolytic cells requires the generation of ROS controlled by the activity of mitochondrial $\mathrm{H}^{+}$-ATP synthase," Carcinogenesis 27(5), 925-935 (2006).

34. T. S. Chen, X. P. Wang, L. Sun, L. X. Wang, D. Xing, and M. Mok, "Taxol induces caspase-independent cytoplasmic vacuolization and cell death through endoplasmic reticulum (ER) swelling in ASTC-a-1 cells," Cancer Lett. 270(1), 164-172 (2008).

35. L. X. Wang, T. S. Chen, J. L. Qu, and X. B. Wei, "Photobleachingbased quantitative analysis of fluorescence resonance energy transfer inside single living cell," J. Fluoresc. 20(1), 27-35 (2009).

36. R. E. Shackelford, W. K. Kaufmann, and R. S. Paules, "Oxidative stress and cell cycle checkpoint function," Free Radic Biol. Med. 28(9), 1387-1404 (2000)

37. N. Zamzami, P. Marchetti, M. Castedo, D. Decaudin, A. Macho, T. Hirsch, S. A. Susin, P. X. Petit, B. Mignotte, and G. Kroemer, "Sequential reduction of mitochondrial transmembrane potential and generation of reactive oxygen species in early programmed cell death," J. Exp. Med. 182(2), 367-377 (1995).

38. D. Safiulina, V. Veksler, A. Zharkovsky, and A. Kaasik, "Loss of mitochondrial membrane potential is associated with increase in mitochondrial volume: physiological role in neurones," J. Cell Physiol. 206(2), 347-353 (2006).

39. P. Delivani, C. Adrain, R. C. Taylor, P. J. Duriez, and S. J. Martin, "Role for CED-9 and Egl-1 as regulators of mitochondrial fission and fusion dynamics," Mol. Cell 21(6), 761-773 (2006).

40. X. J. Jiang and X. D. Wang, "Cytochrome $c$ promotes caspase-9 activation by inducing nucleotide binding to Apaf-1," J. Biol. Chem. 275(40), 31199-31203 (2000).
41. J. F. Lovell, L. P. Billen, S. Bindner, A. Shamas-Din, C. Fradin, B. Leber, and D. W. Andrews, "Membrane binding by tBid initiates an ordered series of events culminating in membrane permeabilization by Bax," Cell 135(6), 1074-1084 (2008).

42. O. S. Breathnach, B. Freidlin, B. Conley, M. R. Green, D. H Johnson, D. R. Gandara, M. O'Connell, F. A. Shepherd, and B. E. Johnson, "Twenty-two years of phase III trials for patients with advanced non-small-cell lung cancer: sobering results," J. Clin. Oncol. 19(6), 1734-1742 (2001)

43. K. Aquilano, P. Vigilanza, G. Rotilio, and M. R. Ciriolo, "Mitochondrial damage due to SOD1 deficiency in SH-SY5Y neuroblastoma cells: a rationale for the redundancy of SOD1," FASEB J. 20(10), 1683-1685 (2006).

44. L. Castera, A. S. Hatzfeld-Charbonnier, C. Ballot, F. Charbonnel, E. Dhuiege, T. Velu, P. Formstecher, L. Mortier, and P. Marchetti, "Apoptosis-related mitochondrial dysfunction defines human monocyte-derived dendritic cells with impaired immuno-stimulatory capacities," J. Cell Mol. Med. 13(7), 1321-1335 (2009).

45. H. Puthalakath, C. S. David, D. C. S. Huang, L. A. O’Reilly, S. M. King, and A. Strasser, "The proapoptotic activity of the Bcl-2 family member Bim is regulated by interaction with the dynein motor complex," Mol. Cell 3(3), 287-296 (1999).

46. C. Chen, J. Yan, Q. Sun, L. Yao, Y. Jian, J. Lu, and J. Gu, "Induction of apoptosis by $110 \mathrm{C}$ requires mitochondrial translocation of the proapoptotic BCL-2 family member BAD," FEBS Lett. 580(3), 813821 (2006)

47. G. V. Putcha, S. Le, S. Frank, C. G. Besirli, K. Clark, B. Chu, S. Alix, R. J. Youle, A. LaMarche, A. C. Maroney, and E. M. Johnson Jr., "JNK-mediated Bim phosphorylation potentiates Bax-dependent apoptosis," Neuron 38(6), 899-914 (2003).

48. J. Zhang and G. T. Bowden, "Targeting Bcl-XL for prevention and therapy of skin cancer," Mol. Carcinog. 46(8), 665-670 (2007). 